\title{
Mechanisms for drawdown of floating particles in a laminar stirred tank flow
}

\author{
He Gong ${ }^{a, b}$, Fenglei Huang ${ }^{a, b}$, Zhipeng Li ${ }^{a, b,{ }^{*}}$, Zhengming Gao ${ }^{a, b,{ }^{*}}$, J.J. Derksen ${ }^{c}$ \\ ${ }^{a}$ Beijing Advanced Innovation Center for Soft Matter Science and Engineering, Beijing University of Chemical Technology, Beijing 100029, China
}

${ }^{\mathrm{b}}$ State Key Laboratory of Chemical Resource Engineering, School of Chemical Engineering, Beijing University of Chemical Technology, Beijing 100029, China

${ }^{\mathrm{c}}$ School of Engineering, University of Aberdeen, Aberdeen AB24 3UE, UK

* Corresponding author. Tel.: +8610 64418267; fax: +8610 64449862. E-mail address: lizp@mail.buct.edu.cn (Zhipeng Li), gaozm@mail.buct.edu.cn (Zhengming Gao).

\begin{abstract}
:
Particle image velocimetry (PIV) experiments on a laminar stirred tank flow with floating particles at just drawdown conditions were performed. Careful refractive index matching of the two phases allowed to resolve the flow around the particles. The maximum solids volume fraction was $4 \%$. The impeller was a pitched blade turbine with upward and downward pumping modes and with different off-bottom clearances, and the impeller-based Reynolds number ranged from about 50 to 120 . Computational fluid dynamics (CFD) coupled to a discrete phase model (DPM) and discrete element method (DEM) was used to predict the flow field, with an emphasis on the tangential velocity component. The liquid velocity profiles predicted by the CFD simulations are in good agreement with the PIV experimental data. The drawdown process and local particle accumulation simulated by the DPM-DEM model agrees with the experimental phenomena as well. Tangential velocity and particles collisions around the shaft trigger the onset of the drawdown of the floating particles.
\end{abstract}

Keywords: drawdown; floating particles; particle image velocimetry; refractive index matching; stirred tank

\section{Highlights:}

- Floating particles distribution and liquid velocity were simultaneously measured

- The simulated liquid and particle characteristics agree well with experimental data 
- Tangential velocity and particle collision trigger the drawdown of floating particles

\section{Introduction}

Solid-liquid stirred tanks with floating particles (their densities are less than that of liquid) are commonly used in a variety of industrial processes, such as the production processes of coatings, cosmetics and lubricating oil [1]. While the suspension of solids that are heavier than the surrounding liquid has received extensive attention, limited information is available in the literature on the drawdown of floating particles in stirred tanks, which justifies investigating the mechanisms of particle drawdown in stirred tanks.

Since the first work regarding floating particles by Joosten et al. [2], researchers have focused on the macroscopic parameters including the just-drawdown speed $N_{\mathrm{jd}}[3-10] . N_{\mathrm{jd}}$ is usually defined as the minimum impeller speed where all particles could be drawn down from the surface of the tank within 1-2 seconds, and it characterizes the performance of the mixing system. With this criterion, the effect of the physical properties of liquid and solids as well as solids loading on the $N_{\mathrm{jd}}$ have been considered, and several $N_{\mathrm{jd}}$ correlations were obtained for a variety of mixing configurations [3-5]. Paglianti et al. [11] used an electrical resistance tomography technique to measure solids concentration distributions of floating particles and proposed a new method for estimating the mixing time. Some researchers extended their work to the suspension of floating particles in gas-solid-liquid systems because many mixing systems are aerated in the process industries. In gas-solid-liquid systems, $N_{\text {jd }}$ sometimes decreases as the gas rate increases, or $N_{\text {jd }}$ increases early as the gas rate increases and then decreases after it reaches a maximum value $[12,13]$. Bao et al. $[14,15]$ investigated the effect of the concentrations of floating particles on the $N_{\mathrm{jd}}$ in a three phases stirred tank. 
Laser-based optical measurement techniques such as particle image velocimetry (PIV) and laser Doppler velocimetry (LDV) have been used in experiments to investigate single-phase flows or dilute two-phase flows. Solid-liquid flows present a challenging environment for the application of these optical imaging techniques; the presence of solids makes the two-phase flow opaque and not directly suitable for PIV and LDV measurements. Thus, the maximum solids concentration is usually limited to $1 \%$ by volume. At higher solids loadings, the laser light will more and more likely be obstructed and scattered by the solids. To overcome this problem, many researchers employed refractive index matching (RIM) in experiments [16-19]. Wiederseiner et al. [20] summarized currently available RIM methods. However, the RIM method was rarely reported in a solid-liquid system with floating particles. Based on our previous research on settling particles [21], we propose a new solid-liquid system to achieve RIM in this work. The solids are Polymethyl methacrylate particles, and the liquid is a mixture of sucrose and sodium chloride aqueous solution.

As for the flow field of solid-liquid systems, most published researches focus on settling particles. In our group we measured the overall flow pattern in the presence of floating particles, however, without matching the refractive indices $[22,23]$. Flat cylindrical shape polyethylene particles $(\$ 3.6 \times 2.1 \mathrm{~mm})$ were used as the floating solid material and the results of the PIV experiments show that the mean flow velocity decreases with an increase of particles concentration from $0 \%$ to $1 \%$ by volume [23]. The flow field near the liquid surface where floating particles are actually drawn down and the flow characteristics around particles were not considered in [23]. To facilitate a detailed analysis in draw-down mechanisms, in the current study, we simplify the flow system by placing a lid on top of the liquid surface and by decreasing the Reynolds number to make the flow laminar. In addition, to resolve the flow characteristics in the drawdown process of floating particles by using PIV, much larger spherical particles with diameter $d_{p}=9.6 \mathrm{~mm}$ were used to enhance resolution and the RIM technique was used to allow for an increase of the particle concentration. Details of our experimental setup will be discussed in the next section. 
Computational fluid dynamics (CFD) has been used extensively to predict solids suspension in stirred tanks with the solids denser than the liquid (settling solids). However, only a limited set of CFD studies on floating particles in stirred tanks is available [24-29]. The Eulerian-Eulerian multiphase model along with the standard $k-\varepsilon$ mixture turbulence model were usually used in these simulations and macroscopic parameters, such as flow pattern, power number, and solid concentration, have been investigated. Based on experimental and simulated results, some controlling factors, including surface vortex formation, turbulent fluctuations, and mean drag, have been identified [26-29]. However, particle collision was always neglected in these simulations. Recently, Li et al. [30] found that particle collision has a significant effect on the solid-liquid flow even when solids volume fractions are very small (about $O\left(10^{-4}\right)$ ). At present, research on simulations of floating particle characteristics in stirred tanks by considering particle collision has not been reported.

The aim of this paper is in the first place to show the feasibility of particle-resolved PIV experiments with floating particles in a laminar flow. We constructed this base-case so as to address some fundamental mechanisms for the drawdown of floating particles and will gradually add complexity such as turbulent flow in the future. The tank-averaged solids volume fraction is up to $4 \%$. Local accumulation phenomena of the solids around the top of the shaft can be observed. In the second place, by comparing experimental and simulated flow fields near the impeller and the shaft, we validate our simulation results with the DPM-DEM model and assess their accuracy. The effect of impeller pumping modes, off-bottom clearance, and solids concentrations on the flow dynamics are discussed as well. In the third place, based on analyses of the tangential velocity and pressure distributions and the just drawdown process simulated with collision models, we can draw conclusions regarding the mechanism of the drawdown of floating particles.

The paper is organized as follows: in the next section, the experimental setup is discussed, including the flow system, PIV experiments and image analysis. Then, the numerical approaches are briefly summarized with references to the relevant literature. In the subsequent Results section we first present the phenomena of the just 
drawdown of floating particles from the surface. Secondly, we discuss the effects of impeller pumping mode,

off-bottom clearance, and overall solids loading on the liquid flow field. Thirdly, we compare the simulation results with the PIV experimental data, and identify the just drawdown conditions. The final section provides a summary and conclusions.

\section{Experimental setup}

\section{Flow system}

A schematic view of the flow geometry is shown in Fig. 1. A cuboid glass stirred tank with a flat square base ( $T \times T=0.22 \times 0.22 \mathrm{~m}^{2}$ ) was used and the liquid height $H$ is equal to $T$. In order to construct a simplified drawdown of floating particles, a lid was placed on the top of the liquid, thus, the floating particles are entirely wetted without trapped air in the drawdown and the free surface is replaced by a no-slip wall, which also is convenient for simulating the boundary. A 4-blade $45^{\circ}$ pitched-blade turbine (PBT) with diameter $D=0.158 \mathrm{~m}$ was operated at three off-bottom clearances $(C=T / 3, T / 2$, and $2 T / 3)$ and two impeller pumping modes (upward and downward pumping), see Table 1.

Polymethyl methacrylate (PMMA) spherical particles were selected as the dispersed phase because of their good transparency and density $\left(\rho_{p}=1210 \mathrm{~kg} / \mathrm{m}^{3}\right)$. The diameter of each PMMA particle in the experiments was measured with an electronic vernier caliper, and the average diameter $d_{p}$ is $9.6 \mathrm{~mm}$ with a standard deviation of 0.15 $\mathrm{mm}$.

To match the refractive index of the PMMA particles $\left(n=1.487\right.$ at $25.0^{\circ} \mathrm{C}$ ), a mixture of sucrose and sodium chloride aqueous solution ( $66.5 \%$ sucrose, $8.87 \%$ sodium chloride, and $24.63 \%$ water by weight) was made as the continuous phase. The refractive index of the mixture was measured with a WAY-2W Abbe refractometer (Shanghai INESA Instrument, China) and the dynamic viscosity with a MARS40 Rheometer (Haake, Germany). Both the refractive index and the dynamic viscosity of the continuous phase are sensitive to temperature (Fig. 2), and therefore the operating temperature was controlled at a fixed temperature of $25.0^{\circ} \mathrm{C}$ in our experiments. At this temperature, the refractive index of the continuous phase is $n=1.481$ and the dynamic viscosity is $\mu=1.365 \mathrm{~Pa} \cdot \mathrm{s}$. The fluctuations of the liquid temperature were within $\pm 0.5^{\circ} \mathrm{C}$ so that the variation of the refractive index and the 
viscosity are within $0.2 \%$ and $6 \%$ of their nominal values respectively. Figure 3 shows the images of a ruler behind a PMMA particle submerged in different liquids. Good refractive index matching avoids light distortion and makes us clearly see the information inside the stirred tank. The density of the continuous phase is $1410 \mathrm{~kg} / \mathrm{m}^{3}$, and the density ratio of the solid and liquid phases $\rho_{p} / \rho=0.858$.

Although we carefully check and control the experimental conditions such as particle quality and liquid temperature, it is difficult to achieve perfect refractive index matching. The liquid mixture was close to being saturated and crystallization sometimes occurred in the experiments which then made the liquid opaque and changed its refractive index as well. Since the liquid viscosity is high, it proved difficult to remove tiny air bubbles. These imperfections somewhat limited the overall solids volume fractions to be considered in this study: we went up to $4 \%$ (920 floating particles), whereas in previous work [21] we could - with a different solid-liquid combination reach $8 \%$.

The just drawdown speed was determined by using an observation method with the criterion that no floating particles remain at the top surface for more than 2 seconds. Instead of monitoring the whole top surface, we only observed several regions where the particle drawdown was difficult, such as the regions near shaft or corner. During the experiments, the impeller speed was gradually increased with an interval of about $5 \mathrm{rpm}$ (revolutions per minute) until the just drawdown condition was reached. For example, the critical just drawdown condition was reached at $N_{\mathrm{jd}}=143.8 \mathrm{rpm}$ in Case 1 (see Table 1 ), but the condition was not met at $N=139 \mathrm{rpm}$. The impeller speed was controlled by a frequency converter (Danfoss, Denmark). Speed fluctuations were less than $0.1 \mathrm{rpm}$. Each experiment has been performed at least two times to check the reproducibility of $N_{\mathrm{jd}}$. The impeller Reynolds number is defined as $R e=N D^{2} / v$ with $N$ impeller speed (in rev/s), $D$ impeller diameter, and $v$ the kinematic viscosity of liquid. For the current experiments, Re ranges from about 50 to 120. The Shields number $\theta \equiv \frac{\rho N^{2} D^{2}}{g d_{p}\left(\rho-\rho_{p}\right)}$ (with g gravitational acceleration and $N$ in rev/s) ranges from about 7 to 42 (see Table 1); it expresses the competition between inertial stresses $\rho N^{2} D^{2}$ and net gravity $g d_{p}\left(\rho-\rho_{p}\right)$.

\section{PIV experiments}


The 2D-PIV system used in this work is a commercial system from TSI (USA). It consists of a $532 \mathrm{~nm} 200 \mathrm{~mJ}$ Nd:YAG dual pulse laser (Beamtech, China), spherical and cylindrical lenses which transform a laser beam into a laser sheet with thickness of $1 \mathrm{~mm}$ at the measurement plane, a 4008×2672 pixels charge coupled device (CCD) camera (TSI, USA), a synchronizer (TSI, USA), a shaft encoder (Kubler, Germany), and Insight 3G software. The transistor-transistor logic (TTL) signals generated by the shaft encoder were transferred to the synchronizer to synchronize the blade position, the laser pulses, and the image capture, thus, impeller angle-resolved measurements were performed. The impeller angle between the impeller blade and the measurement plane is defined as $\theta$, see Fig. 1. Figure 4a shows the effect of the impeller angle on the mean flow field. Similar to results in the literature [31], the flow field in impeller discharge region is closely associated with the impeller positions. Away from the impeller, its influence on the flow field gradually decreases. The impeller angle has very weak influence on the mean velocities near the surface of the tank, see Fig. 4b. Since the main region of interest is the region near the top surface, we only present flow fields at $\theta=0^{\circ}$ in the following sections. Hollow spherical glass particles (TSI, USA) with diameters of about $8-12 \mu \mathrm{m}$ and density of $1500 \mathrm{~kg} / \mathrm{m}^{3}$ were used as PIV tracer particles.

The $(x, y, z)$ coordinate system is shown in Fig. 1 . The Cartesian coordinates $x, y$, and $z$ in the measurement plane correspond to radial, tangential, and axial direction, respectively, and the mean velocities in the three directions are represented by $U, V$, and $W$, respectively. The origin of the coordinate system is the center of the tank bottom. The PIV measurement area was located between the impeller discharge region and the top wall in axial direction and is in the $x-z$ plane, as illustrated by the blue rectangle and green line in Fig. 1.

To obtain the instantaneous velocity field, the PIV raw images were divided first into interrogation areas of $48 \times 48$ pixles $^{2}$ with $50 \%$ overlap by using the Nyquist grid combined with the ZeroPad mask method [32]. Then, the interrogation windows were cross-correlated with the fast Fourier transform algorithm, and the vectors were calculated with the Gaussian subpixel estimator [33]. The image resolution was $41.49 \mu \mathrm{m} / \mathrm{pixel}$, thus the velocity vector resolution was $0.99 \mathrm{~mm}$, that is, there are about $9-10$ velocity vectors per particle diameter. Two different 
time intervals between the laser pulses were used for each experiment to enhance the accuracy of the PIV results. A short time interval is used for resolving the flow field in the impeller discharge region, while a longer time interval is used for measuring the flow near surface; the time intervals have been specified in Table 1 as $\Delta t_{1}$ and $\Delta t_{2}$ respectively. Within each time interval, the maximum in-plane and out-of-plane displacements of seeding particles were less than one-quarter of the size of the interrogation areas and of the thickness of the laser sheet. The two measurements were combined to generate one final flow field. The statistical convergence of the velocities was verified and is partly shown in Figs. 4c and 4d. One hundred pairs of raw PIV images show converged mean velocities, so that the number of samples in each experimental case in Table 1 is 100.

\section{Detection of spheres}

Figure 5a shows part of a PIV raw image with three particles. The laser sheet went through the particles, forming three circular particle boundaries. Ideally, inside the circular boundaries, no tracer particle image could be found. To detect the circles (i.e. the particles) in the raw images automatically, a Matlab (Mathworks, USA) code was programmed by using a Circular Hough Transform (CHT) algorithm [34] along with a number of pre- and postprocessing steps. The procedure is as follows:

First, the raw PIV images were converted to gray images and the image intensity was adjusted with the Matlab function imadjust to enhance the contrast. Then the Matlab function imfindcircles was used to perform the CHT. According to our previous experience [21], the parameters in the imfindcircles were tested and validated. The Sensitive factor was set to 0.953 , and the Edge gradient threshold to 0.07. The Object polarity was set to "Bright", and the computation method to "PhaseCode". A sample of the particle boundary detection is shown in Fig. 5b. After deleting the velocity vectors inside the circular particles (Fig. 5c) which were calculated automatically by the Insight 3G software, we will obtain the final instantaneous flow field (Fig. 5d). The deleted velocities due to the presence of the floating particles are not considered in calculating the mean flow velocity so that the mean interstitial velocity is 
obtained. The flow resolution of about nine velocity vectors per particle diameter is illustrated and the interaction between the particles and their surrounding flow can be observed in Fig. $5 \mathrm{~d}$ as well.

\section{Numerical simulations}

\section{Eulerian-Lagrangian approach with DPM-DEM models}

The numerical simulations were performed based on the commercial software package Fluent 18.0 [35]. The Lagrangian discrete phase model (DPM) follows the Eulerian-Lagrangian approach in Fluent. The fluid phase is treated as a continuum by solving the volume-averaged Navier-Stokes equations in terms of the interstitial liquid velocity [36]. The solid phase is solved by tracking a large number of particles in the calculated flow field. The discrete phase exchanges momentum with the fluid phase.

In the DPM modeling, the motion of the discrete phase is treated in Lagrangian terms, i.e. by solving the force balance on the particles:

$$
\frac{d \vec{u}_{p}}{d t}=\frac{\vec{u}-\vec{u}_{p}}{\tau_{r}}+\vec{g} \frac{\rho_{p}-\rho}{\rho_{p}}+\vec{F}
$$

where $\tau_{r}=\frac{\rho_{p} d_{p}^{2}}{18 \mu} \frac{24}{C_{d} \operatorname{Re}}$ is the particle relaxation time, $\frac{\vec{u}-\vec{u}_{p}}{\tau_{r}}$ is the drag force per unit particle mass, $\vec{F}$ is the sum of additional forces including the force caused by collisions among particles. The drag coefficient $C_{d}$ is calculated based on the correlations proposed by Morsi and Alexander [37].

The discrete element method (DEM), which was first proposed by Cundall and Strack [38], accounts for the forces that result from the collisions of particles. For a mono-sized spherical particle, the spring-dashpot collision law in the DEM is as follows:

$$
\vec{F}_{1}=-\vec{F}_{2}=\left[K \delta-\ln \eta \sqrt{\frac{2 K m}{\pi^{2}+\ln ^{2} \eta}}\left(\vec{u}_{2}-\vec{u}_{1}\right) \cdot \vec{e}_{12}\right] \vec{e}_{12}
$$


where $K$ is a spring constant, $\delta$ is the particle overlap, $\eta$ is a coefficient of restitution for the dashpot term, $\mathrm{m}$ is the particle mass, $\vec{u}_{1}$ and $\vec{u}_{2}$ are the velocities of particle 1 and particle 2 , respectively, and $\vec{e}_{12}$ is the unit vector pointing from particle 1 to particle 2. To save computational resources, a procedure for detecting collision partners involving a coarse grid was applied. Momentum exchange between the discrete and the continuous phase is computed by examining the position of every particle and the hydrodynamic force it experiences as it passes through each control volume. The forces are then fed back to the volume-averaged Navier-Stokes equations. This way, DPM coupled with DEM realizes two-way coupling between continuous phase and discrete phase, as well as collisions between particles.

\section{Computational aspects}

The laminar flow model was used to calculate the flow field of the continuous phase. The DPM with two-way coupling was used to model the movement of the floating particles. The DEM was chosen to realize the collisions between the floating particles. The impeller rotation was modeled by using the multiple reference frames approach. No-slip condition was applied to all solid wall boundaries.

The geometric parameters used for the simulations were the same as those described in the PIV experiment section. A grid sensitivity study has been carried out and the results will be listed in the next section. The final computational grid consisted of about 1.23 million and 1.77 million unstructured cells in the rotating and the stationary zones, respectively. In the impeller region, a fine grid was utilized with a grid spacing of the order of 1 $\mathrm{mm}$.

To provide good initial values for the transient simulation for the motion of floating particles, a steady state single-phase simulation was conducted first. Then the particles were released in the flow field, and the simulation was switched to transient mode. The spring-dashpot constants $K$ and $\eta$ in the DEM are $1000 \mathrm{~N} / \mathrm{m}$ and 0.9 , respectively. The time step was calculated according to the criterion ( $\Delta t \leq \frac{1}{10} 2 \pi \sqrt{\frac{m}{K}}$ ) proposed by Liu et al. [39] and was set at $10^{-4} \mathrm{~s}$. The value of the spring constant $K$ is a compromise between having sufficiently rigid particles and a reasonably sized (not unpractically small) time step. In solid-liquid systems most dissipation related to particles 
takes place in the liquid, not so much in particle-particle collisions so that the choice of $\eta$ is not that critical. The second order upwind scheme was used for the spatial discretization of the momentum equations, and the second-order implicit scheme for time advancement. Convergence per time step was achieved when the normalized residuals of the continuity and velocities became less than $10^{-4}$. The time step of the DEM process was the same as the one used for the flow simulation. The torque acting on the impeller was monitored as a way of checking when a quasi-steady state was reached.

\section{Results and Discussion}

\section{Phenomena of particle drawdown from top surface}

The drawdown process of the floating particles is shown in Fig. 6 by having two green particles among the further transparent particles. The overall solids volume fraction is $\phi=1 \%$ (Case 1, 230 floating particles). The right green particle moves slowly and could be considered for reference. The left one gradually moves to the shaft, rotates around the shaft, descends from the liquid surface to the impeller, participates in the main flow circulation, and then goes back to the surface. This process is quite different from previous results in the literature. For example, Özcan-Taşkin et al. [28] found that the drawdown position of floating particles is close to the vessel walls when a downward pumping impeller is mounted close to the vessel base, and Liu et al. [40] found that an eccentric vortex is able to generate a strong vertical force to draw down floating particles. The main reason for the difference in drawdown positions and patterns is that the investigated flow conditions vary greatly; the flow fields in the literature are mainly turbulent, while they are laminar in this study. Another reason might be the squared shape of the tank.

The left panel of Fig. 7 shows a PIV raw image near the shaft and the lid. Particles accumulate around the shaft and are being drawn down, forming an inverted conical shape. After detecting the particles and deleting the vectors inside the particles, we obtain the instantaneous flow field in this region, as shown in the right panel of Fig. 7. As can be seen, in the conical region the liquid velocity is very weak as the particles are mostly in contact with each other. Outside this region an overall recirculation generated by the impeller and modified by the accumulated particles pushes particles to the shaft and draws them down. 


\section{Effect of impeller pumping mode, clearance, and solid concentration}

The left and middle panels of Fig. 8 show the mean velocity field of the continuous phase for the upward and downward impeller pumping modes at just-drawdown conditions. As expected, the impeller discharge stream, the circulation region, and the velocity distribution at the two pumping modes are different in the region between the impeller and the lid. Besides the main circulation, a secondary circulation forms above the impeller for the upward pumping mode, while only one circulation could be found for the downward one. In turbulent flows [26, 41], a mostly axial flow pattern is observed in the impeller discharge region. In laminar flows -as in this study - the axial flow is suppressed, and radial flow dominates the impeller discharge region.

The axial velocity profiles at two horizontal lines, $z / H=0.89$ and $z / H=0.93$, are shown in the right panel of Fig. 8 . We present these velocities in dimensional form since the two situations (upward pumping, downward pumping) are both at just-drawdown conditions. We observe that for the two pumping modes the vertical velocities near the top surface are quite different. From Table 1, we find that the $N_{\mathrm{jd}}$ for the upward pumping model is larger than that for the downward pumping mode, which means that it is more efficient (with a smaller impeller speed and thus lower power input) for the latter mode to draw down the floating particles in a laminar flow.

The mean velocity fields of the continuous phase for three impeller clearances are presented in the left three panels of Fig. 9. As the impeller off-bottom clearance decreases, the just drawdown speed increases from $N_{\text {jd }}=121.3$ rpm at $C=2 T / 3$ to $N_{\mathrm{jd}}=285.5 \mathrm{rpm}$ at $C=T / 3$, thus the velocity magnitude increases dramatically at $C=T / 3$ in the impeller region. With the decrease of the clearance (or the increase of the impeller submergence), only one circulation forms in the region above the impeller, and the shape of the circulation changes from circular to elliptical. A qualitative comparison in terms of velocity contour shows that the velocity distribution is similar near the liquid surface. The axial velocity profiles at two horizontal lines is shown in the very right panel of Fig. 9, which quantitatively confirms that almost the same axial velocity profiles are needed to draw the floating particles down near the shaft. At $C=T / 3$ the discrepancy might be caused by the dramatic change of the circulation shape and higher power input (because a 
larger impeller speed was required). This means that an impeller operating at very small off-bottom clearance is not an energy-efficient design.

The left four panels of Fig. 10 show the mean velocity field of the continuous phase for four solids concentrations at $C=T / 2$ and downward pumping mode. With the increase of solids concentration from $1 \% v / v$ to $4 \%$ $v / v$, the just drawdown speed increases from $N_{\mathrm{jd}}=143.8 \mathrm{rpm}$ to $197.4 \mathrm{rpm}$, that is, it is necessary to input more energy to draw down the increased number of floating particles. A single-phase flow field with $N=197.4 \mathrm{rpm}$ is also shown in Fig. 10e. This allows us to directly assess the effects the particles have on the overall liquid circulation pattern in the tank (compare Figure 10d and 10e). The single-phase impeller outstream is much stronger than when solids are present. This makes that the circulation extends to higher up in the mixing tank. The impact of the high concentration of particles in the corner between lid and shaft on the liquid flow is also clearly visible with very weak flow there in the two-phase system. These observation are further specified in Figure 11 (compare results at $0 \%$ and $4 \%$ solids), with the single-phase impeller outstream stronger than its two-phase counterpart (top panels of Figure 11) and higher (in absolute sense) flow velocities in the upper regions of the tank for the single-phase system. These findings agree with our previous results in a turbulent flow; both the average velocities and the turbulent intensities are weakened by the presence of settling particles [21].

In the top row of Fig. 11, the radial velocity peak in the impeller region increases as the solids concentration increases from $1 \% v / v$ to $4 \% v / v$. As discussed in the last paragraph, the more particles, the more attenuation of the flow field. Thus, the velocity peak increase is caused by the increase of impeller speed, which was used to draw down the additional particles. Dimensionless velocity profiles scaled with the impeller tip velocity will present different velocity distribution because the impeller speeds are different (see Table 1). We use dimensional velocities in this work in order to characterize the critical condition to drawn down the floating particles. From the bottom row of Fig. 11, we can confirm that almost the same axial velocity profiles are achieved when the horizontal lines approach the liquid surface. 


\section{Comparisons of numerical and experimental results}

Figure 12 shows the experimental and simulated velocity fields of the single-phase flow. Both the main circulation and the secondary flow below the impeller can be well predicted by the simulation. The simulated impeller discharge stream, however, moves vertically for a slightly longer distance along the side wall of the tank. The attenuation of the predicted velocity magnitude is a little faster than that of the experimental data, particularly in the top part of the tank. To quantify the discrepancy between experimental and simulated results, we present the radial and axial velocity profiles at one vertical line, $x / T=0.45$, and one horizontal line, $z / H=0.93$, in Fig. 13 . The radial velocity in the vicinity of the impeller, as well as the axial velocity in the top part of the tank are overpredicted. The radial profile at $x / T=0.45$ shows the above-mentioned longer movement of the simulated flow along the side wall is caused by the underpredicted radial velocity, as shown in bottom left panel of Fig. 13. The velocity profiles in Fig. 13 also confirm that our simulated results with 1.5 million cells is already grid insensitive with the results from the three grids overlapping in Fig. 13.

The simulated solid-liquid flow with solid concentration $4 \%$ is compared with the corresponding experimental data in Figs. 14 and 15. Although there are some discrepancies, the simulated results are in good agreement with experimental data overall, which gives us confidence that we can use the simulations for investigating drawdown mechanisms. It is encouraging to see that weak flow due to the presence of particles in the corner between lid and shaft as observed in the experiment is captured well by the simulations. This shows the two-way coupling as contained in the numerical procedure. In a quantitative sense (see the velocity profiles in Figure 15) the agreement between simulation and experiment is excellent. This is to some extent expected given the laminar nature of the flow.

Figure 16 shows the experimental and simulated solid volume fraction contours and profiles. The simulated particle accumulation around the top of the shaft agrees with the experimental data. Particles first accumulate near the lid after they circulate back from the main flow, then they move to the shaft, forming a conical region where 
particles are in sustained contact with each other. The predicted average solids volume fraction at $z / H=0.90$ is slightly larger than the experimental one in the conical region. But in other regions such as the circulation and the impeller zones, the averaged solid volume fraction is underpredicted in the simulation. One reason might be that the actual shape of the particles and the volume they occupy are not considered in the current DEM model. Another reason might be that particle rotation was neglected in the simulation; particle rotation is not compatible with simulations using the multiple reference frames method in Fluent.

The role of the radial velocity is to pull the particles toward the shaft; it is not directly helpful for particle drawdown. The profiles of the axial velocity gradually tend to collapse at different impeller clearance, as well as various solids concentrations (see Figs. 9 and 11). The axial velocity around the shaft could pull down the floating particles, but it is very weak, which means that axial velocity is not the main factor for dawning down the floating particles. The simulated tangential velocities for different solids concentrations tend to collapse to one curve at $z / H=$ 0.93 in the region near the shaft $(0<x / T<0.1)$, and its values are much larger than the axial and radial velocities in this region, as shown in Fig. 17. Thus, we consider that the tangential velocity could play a role in the drawdown mechanism of the particles.

To address how tangential velocity could promote particle drawdown we realize that tangential velocity through centrifugal effects - induces pressure gradients. Therefore, the normalized pressure distribution near the shaft region is presented in Fig. 18. There we see two regions with low pressure: one is near the lid, and another is near the shaft. The upper low-pressure region promotes particle accumulation. If a particle moves towards the lower low pressure region, it will be drawn down very quickly, and then move along the shaft (as could be seen in Fig. 6). The net gravity force on one floating particle is $0.0009 \mathrm{~N}$. If we assume that a pressure gradient is responsible for the particle drawdown, the critical pressure difference to draw down the particle is $\Delta p=0.0009 /\left(\frac{1}{4} \pi d_{p}^{2}\right)=12.44$ $\mathrm{Pa}$, and then the normalized pressure coefficient is $c_{p}=\Delta p /\left(\frac{1}{2} \rho V_{\text {tip }}^{2}\right)=0.0066$. Given the pressure differences 
on the scale of the particle size as can be estimated from Fig 18, the low pressure region near the shaft is capable of drawing down the particles. As can be seen in the right panel of Figure 18, the presence of the particles changes the pressure distribution in comparison with the pressure field of the single-phase flow, which means that the interaction between the particles and liquid are represented in the simulations.

The initial positon of the particles in the simulation with $\Phi=4 \%$ is shown in the left panel Fig. 19. After about 20 impeller revolutions, particles are continuously being drawn down from the region around the shaft and then going back the top and surface region. A snapshot of this process is presented in the middle panel of Fig. 19. In our simulations, we found that the DEM model must be used together with the DPM model to correctly predict the just-drawdown process, otherwise, none of the floating particles could be drawn down (see the right panel of Fig. 19). Thus, the collisions among the particles is another key factor for the just drawdown of the floating particles.

\section{Conclusions}

Refractive index matching between floating particles and the liquid phase was used to achieve the measurement of liquid velocity and particle positions simultaneously with our 2D-PIV system. The maximum solids concentration by volume is $4 \%$. A matlab code was programmed to detect the in-plane particle boundaries and to delete the erroneous vectors inside the boundaries and then to obtain the instantaneous flow fields. To the best of our knowledge, this is the first work regarding simultaneously measuring the liquid velocity and particles concentration in a solid-liquid system with floating particles.

The snapshot of painted particles and the instantaneous PIV results show that the floating particles accumulate around the shaft while they are being drawn down along the rotating shaft. Particles collect in an inverted conical shape near the shaft before being drawn down. In this region, the in-plane liquid velocity is attenuated as the solids concentration is locally very high. 
To quantify the flow field in under just-drawdown conditions, we checked the velocity transfer process from the impeller to the near shaft region at different operating conditions, including two pumping modes, three off-bottom clearances, and four solids concentrations. The axial velocities that could draw down the particles almost overlap each other near top surface for some operating conditions. However, the values of the axial velocities are small and are getting close to zero near the shaft.

The axial and radial velocities predicted by the CFD simulations are validated by the experimental data, and good agreement is obtained. Compared with the axial and radial velocity fields, the pressure induced by the tangential velocity distribution around the shaft is a main factor to draw down the floating particles. The instantaneous particle positions simulated by the Eulerian-Lagrangian approach with the DPM-DEM model confirm that particle collision around the shaft is an additional mechanism for drawing down the particles.

In this study we constructed a simplified laminar flow system and omit some complexities of the free surface to investigate the characteristics of the just drawdown of floating particles. The experimental data will enrich the existing data sets for validating computational methods and models. Gradually adding complexity, such as increasing the Reynolds number to make the liquid flow transitional or turbulent, and removing the top lid to consider the free surface, will be our future research directions. Also more work regarding the Eulerian-Lagrangian approach with DPM-DEM models, as well as the Eulerian-Eulerian approach with different models, should be considered in future research.

\section{Acknowledgement}

The authors gratefully acknowledge the financial support from the National Key R\&D Program of China (2017YFB0306701) and from the National Natural Science Foundation of China (No.21676007).

\section{References}

[1] S. Kondo, N. Yamada, K. Takahashi, Distribution of solid particles lighter than liquid in an agitated vessel stirred by dual impellers, J. Chem. Eng. Jpn. 41 (2008) 155-160. 
[2] G.E.H. Joosten, J.G.M. Schilder, A.M. Broere, The suspension of floating solids in stirred vessels, Chem. Eng. Res. Des. 55 (1977) 220-222.

[3] K. Takahashi, S. Sasaki, Complete drawdown and dispersion of floating solids in agitated vessel equipped with ordinary impellers, J. Chem. Eng. Jpn. 32 (1999) 40-44.

[4] N. Kuzmanic, M. Akrap, A. Kovacevic, An experimental investigation into the complete drawdown of floating solids in dual-impeller stirred vessels, J. Chem. Eng. Jpn. 39 (2006) 932-939.

[5] R.W. Thring, M.F. Edwards, An experimental investigation into the complete suspension of floating solids in an agitated tank, Ind. Eng. Chem. Res. 29 (1990) 676-682.

[6] N. Kuzmanić, R. Žanetić, M. Akrap, Impact of floating suspended solids on the homogenisation of the liquid phase in dual-impeller agitated vessel, Chem. Eng. Process. 47 (2008) 663-669.

[7] O. Khazam, S.M. Kresta, A novel geometry for solids drawdown in stirred tanks, Chem. Eng. Res. Des. 87 (2009) $280-290$.

[8] N. Kuzmanić, D. Rušić, Solids concentration measurements of floating particles suspended in a stirred vessel using sample withdrawal techniques, Ind. Eng. Chem. Res. 38 (1999) 2794-2802.

[9] N. Kuzmanić, B. Ljubičić, Suspension of floating solids with up-pumping pitched blade impellers; mixing time and power characteristics, Chem. Eng. J. 84 (2001) 325-333.

[10] K.J. Myers, A.K. Pandit, E.E. Janz, J.B. Fasano, Impeller diameter and submergence effects in solids drawdown with uppumping impellers, Can. J. Chem Eng. 95 (2017) 1076-1081.

[11] A. Paglianti, C. Carletti, A. Busciglio, G. Montante, Solid distribution and mixing time in stirred tanks: the case of floating particles, Can. J. Chem. Eng. 95 (2017) 1789-1799.

[12] S. Xu, W. Ren, X. Zhao, Critical rotational speed for a floating particle suspension in an aerated vessel, Chem. Eng. Technol. 24 (2001) 189-194.

[13] S. Xu, L. Feng, X. Gu, K. Wang, G. Hu, Gas-liquid floating particle mixing in an agitated vessel, Chem. Eng. Technol. 23 (2000) 103-113.

[14] Y. Bao, Z. Gao, Z. Hao, J. Long, L. Shi, J.M. Smith, N.F. Kirkby, Effects of equipment and process variables on the suspension of buoyant particles in gas-sparged vessels, Ind. Eng. Chem. Res. 44 (2005) 7899-7906.

[15] Y. Bao, Z. Hao, Z. Gao, L. Shi, J.M. Smith, Suspension of buoyant particles in a three phase stirred tank, Chem. Eng. Sci. 60 (2005) 2283-2292.

[16] M.A. Abbas, C.T. Crowe, Experimental study of the flow properties of a homogenous slurry near transitional Reynolds numbers, Int. J. Multiphase Flow 13 (1987) 357-364.

[17] Y.A. Hassan, E.E. Dominguez-Ontiveros, Flow visualization in a pebble bed reactor experiment using PIV and refractive index matching techniques, Nucl. Eng. Des. 238 (2008) 3080-3085. 
[18] H. Unadkat, C.D. Rielly, G.K. Hargrave, Z.K. Nagy, Application of fluorescent PIV and digital image analysis to measure turbulence properties of solid-liquid stirred suspensions, Chem. Eng. Res. Des. 87 (2009) 573-586.

[19] A. Gabriele, A.N. Tsoligkas, I.N. Kings, M.J.H. Simmons, Use of PIV to measure turbulence modulation in a high throughput stirred vessel with the addition of high Stokes number particles for both up- and down-pumping configurations, Chem. Eng. Sci. 66 (2011) 5862-5874.

[20] S. Wiederseiner, N. Andreini, G. Epely-Chauvin, C. Ancey, Refractive-index and density matching in concentrated particle suspensions: a review, Exp. Fluids 50 (2011) 1183-1206.

[21] G. Li, Z. Gao, Z. Li, J. Wang, J.J. Derksen, Particle-resolved PIV experiments of solid-liquid mixing in a turbulent stirred tank, AIChE J. 64 (2018) 389-402.

[22] R. Atibeni, Z. Gao, Y. Bao, Effect of baffles on fluid flow field in stirred tank with floating particles by using PIV, Can. J. Chem. Eng. 91 (2013) 570-578.

[23] R. Atibeni, Z. Gao, Y. Bao, PIV investigation of liquid flow field in off-centered shaft stirred tanks with floating particles, Int. J. Chem. React. Eng. 9 (2011) A100.

[24] L. Li, B. Xu, CFD simulation of floating particles suspension in a stirred tank, Chem. Pap. 71 (2017) 1377-1387.

[25] L. Li, B. Xu, CFD simulation of gas-liquid floating particles mixing in an agitated vessel, Chem. Ind. Chem. Eng. Q. 23 (2017) 377-389.

[26] O. Khazam, S.M. Kresta, Mechanisms of solids drawdown in stirred tanks, Can. J. Chem. Eng. 86 (2008) 622-634.

[27] G. Özcan-Taskin, G. McGrath, Draw down of light particles in stirred tanks, Chem. Eng. Res. Des. 79 (2001) 789-794.

[28] G. Özcan-Taşkin, H. Wei, The effect of impeller-to-tank diameter ratio on draw down of solids, Chem. Eng. Sci. 58 (2003) 2011-2022.

[29] G. Özcan-Taşkin, Effect of scale on the draw down of floating solids, Chem. Eng. Sci. 61 (2006) 2871-2879.

[30] Z. Li, J. Wei, B. Yu, Direct numerical study on effect of interparticle collision in particle-laden turbulence, AIAA. J. 54 (2016) 3212-3222.

[31] A. Gabriele, A.W. Nienow, M.J.H. Simmons, Use of angle resolved PIV to estimate local specific energy dissipation rates for up- and down-pumping pitched blade agitators in a stirred tank, Chem. Eng. Sci. 64 (2009) 126-143.

[32] R.D. Keane, R.J. Adrian, Theory of cross-correlation analysis of PIV images, Appl. Sci. Res. 49 (1992) $191-215$.

[33] K.T. Christensen, The influence of peak-locking errors on turbulence statistics computed from PIV ensembles, Exp. Fluids 36 (2004) 484-497.

[34] P.V.C. Hough, Method and means for recognizing complex patterns, US Patent 3069654, 1962.

[35] ANSYS Fluent Theory Guide, ANSYS Inc. 2017. 
[36] J. Capecelatro, O. Desjardins, An Euler-Lagrange strategy for simulating particle-laden flows, J. Comput. Phys. 238 (2013) $1-31$.

[37] S.A. Morsi, A.J. Alexander, An investigation of particle trajectories in two-phase flow systems, J. Fluid Mech. 55 (1972) 193-208.

[38] P.A. Cundall, O.D.L. Strack, A discrete numerical model for granular assemblies, Géotechnique 29 (1979) 47-65.

[39] D. Liu, C. Bu, X. Chen, Development and test of CFD-DEM model for complex geometry: a coupling algorithm for Fluent and DEM, Comput. Chem. Eng. 58 (2013) 260-268.

[40] B. Liu, Y. Zheng, M. Chen, B. Huang, Z. Jin, Experimental study on the mixing and dispersing of floating particles in viscous system, Can. J. Chem. Eng. 94 (2016) 2013-2022.

[41] J. Derksen, Assessment of large eddy simulations for agitated flows, Chem. Eng. Res. Des. 79 (2001) $824-830$. 


\section{Table 1}

Table 1 Operating conditions and just drawdown speeds in the experiments. The particle numbers are 230, 460, 690, and 920 for four solid concentrations $1 \%, 2 \%, 3 \%$, and $4 \%$, respectively.

\begin{tabular}{|c|c|c|c|c|c|c|c|c|}
\hline case & C & $\begin{array}{l}\text { impeller pumping } \\
\text { mode }\end{array}$ & $\begin{array}{l}\text { solids concentration } \\
\qquad(v / v)\end{array}$ & $N_{\mathrm{jd}}(\mathrm{rpm})$ & $R e$ & $\theta$ & $\Delta t_{1}(\mu s)$ & $\Delta t_{2}(\mu s)$ \\
\hline 1 & $T / 2$ & downward & $1 \%$ & 143.8 & 61.80 & 10.75 & 200 & 650 \\
\hline 2 & & & $2 \%$ & 158.9 & 68.29 & 13.12 & 160 & 500 \\
\hline 3 & & & $3 \%$ & 182.7 & 78.52 & 17.35 & 140 & 450 \\
\hline 4 & & & $4 \%$ & 197.4 & 84.84 & 20.25 & 120 & 400 \\
\hline 5 & & & $0 \%$ & 197.4 & 84.84 & 20.25 & 120 & 400 \\
\hline 6 & $2 T / 3$ & & $2 \%$ & 121.3 & 52.13 & 7.65 & 250 & 800 \\
\hline 7 & $T / 3$ & & $2 \%$ & 285.5 & 122.70 & 42.36 & 90 & 300 \\
\hline 8 & $T / 2$ & upward & $2 \%$ & 183.1 & 78.69 & 17.42 & 160 & 500 \\
\hline
\end{tabular}


Figure 1
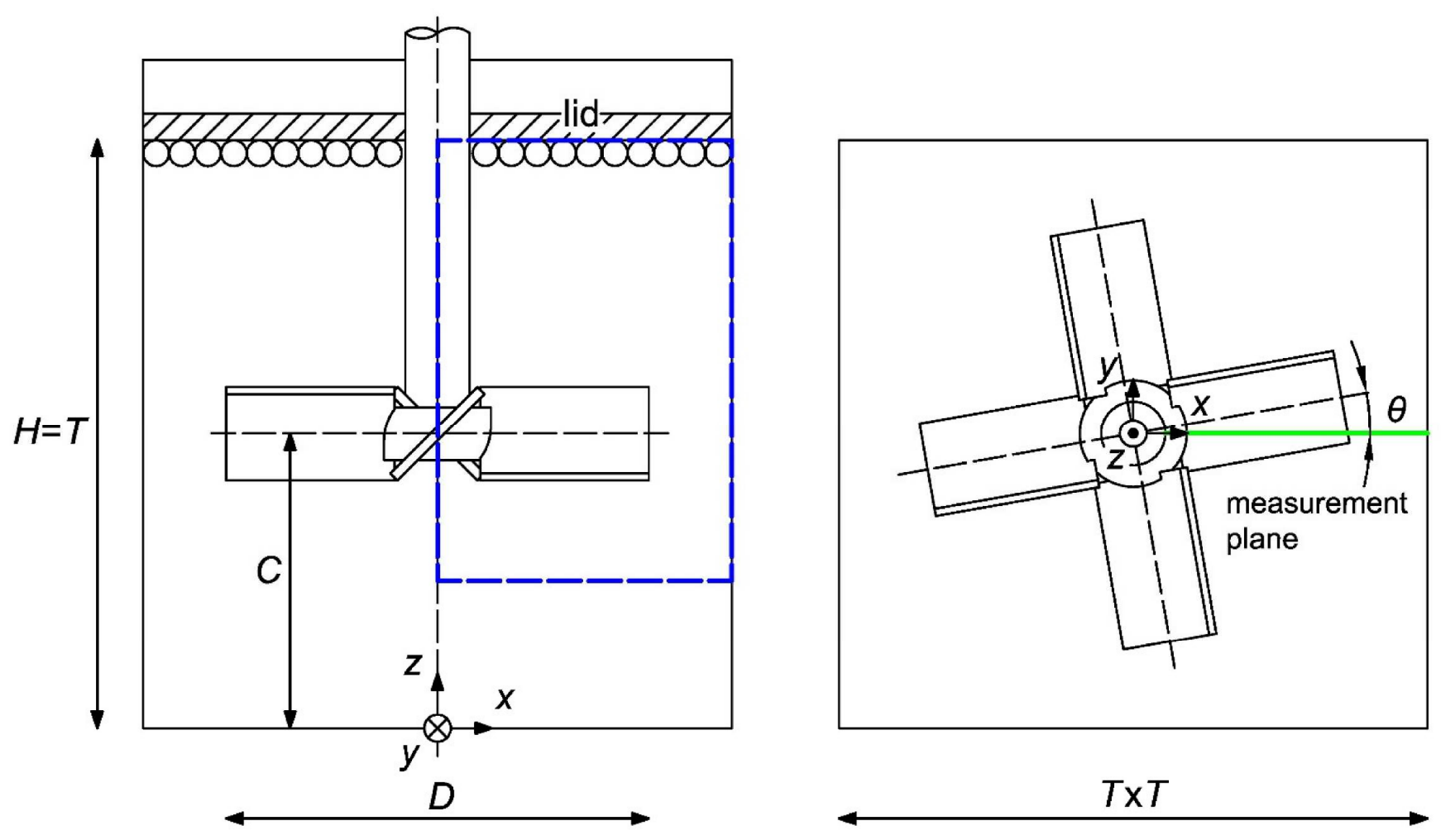

Fig. 1. Stirred tank geometry, $(x, y, z)$ coordinate system, PIV measurement region shown by the blue rectangle, and impeller angle $\theta$ between the measured blade and the measurement plane for downward pumping model. The impeller in the right panel rotates counterclockwise. 


\section{Figure 2}
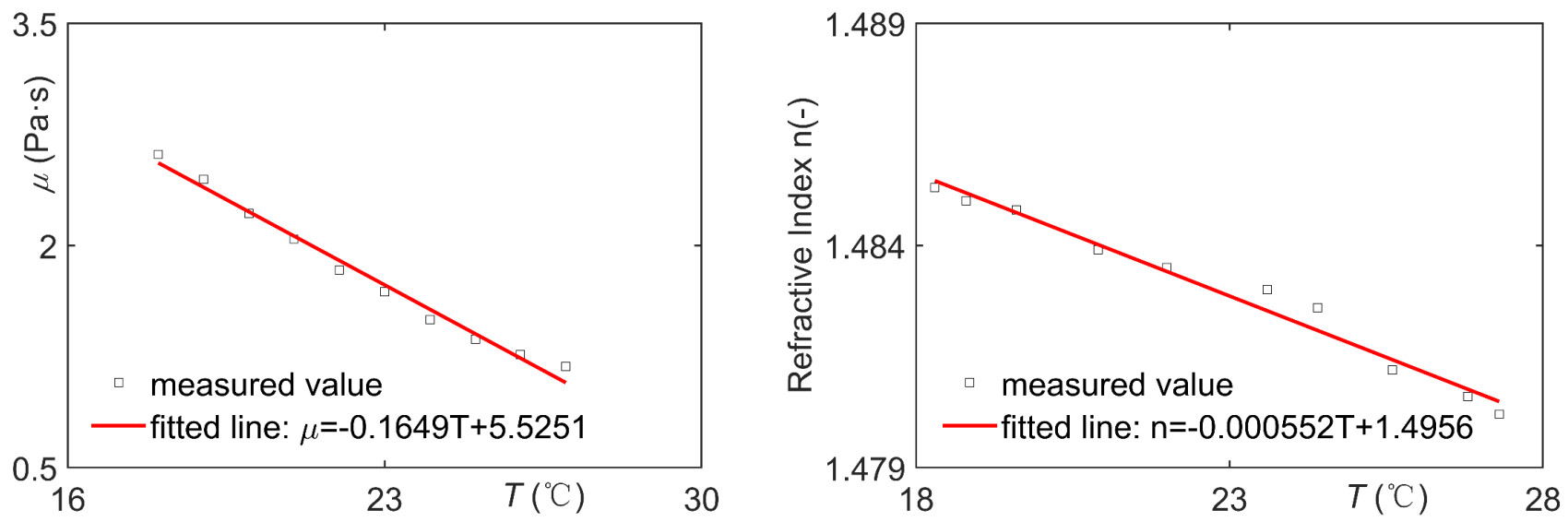

Fig. 2. Dynamic viscosity (left panel) and refractive index (right panel) of the sucrose and sodium chloride aqueous solution used as a function of temperature. $T$ is in ${ }^{\circ} \mathrm{C}$ and $\mu$ in Pa.s in the fitted equations. 


\section{Figure 3}

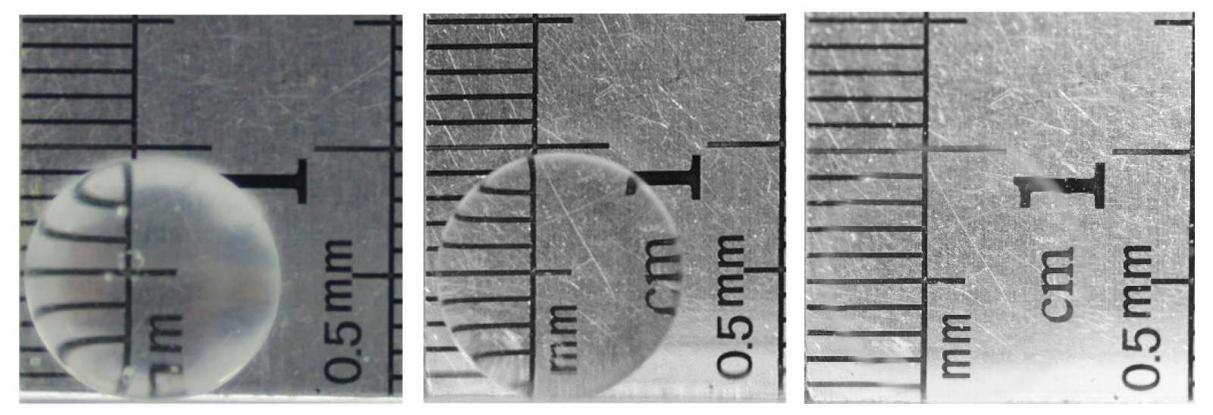

Fig. 3. Refractive index matching images of a PMMA sphere ( $n=1.487)$ immersed in water (left panel, $n=1.3322)$, Phenyl silicone oil (middle panel, $n=1.4610$ ), or the sucrose and sodium chloride aqueous solution (right panel, $n=1.481$ ). 


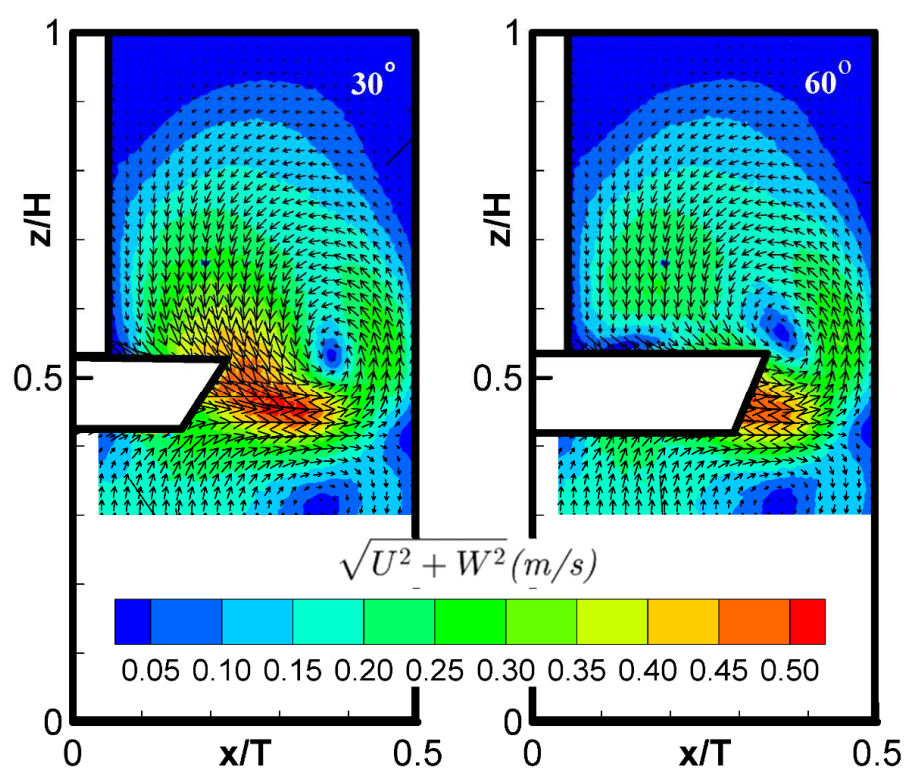

(a)

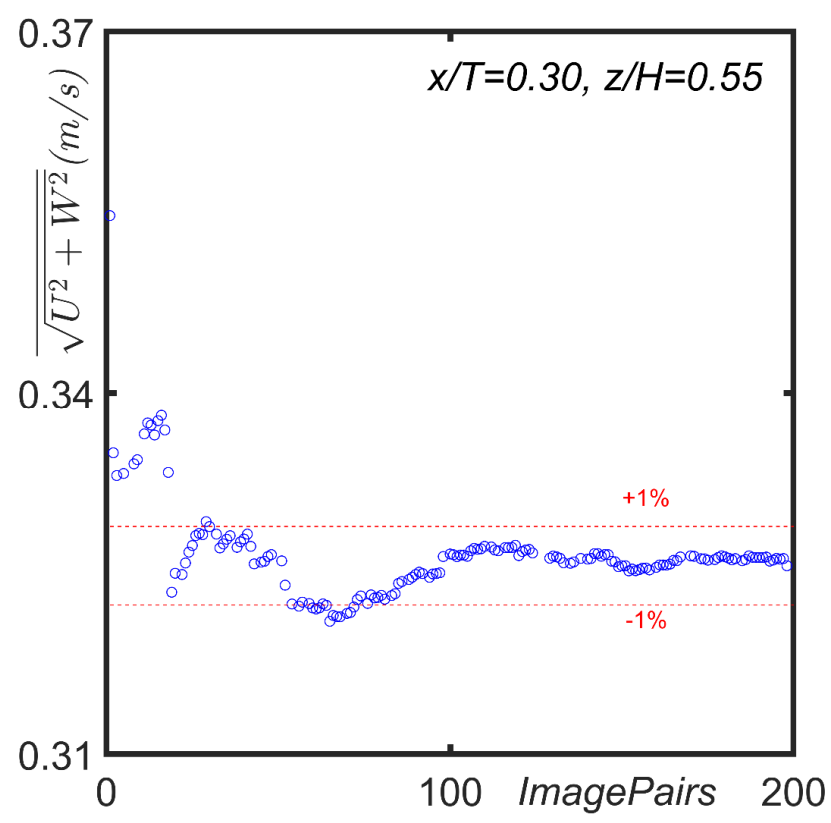

(c)

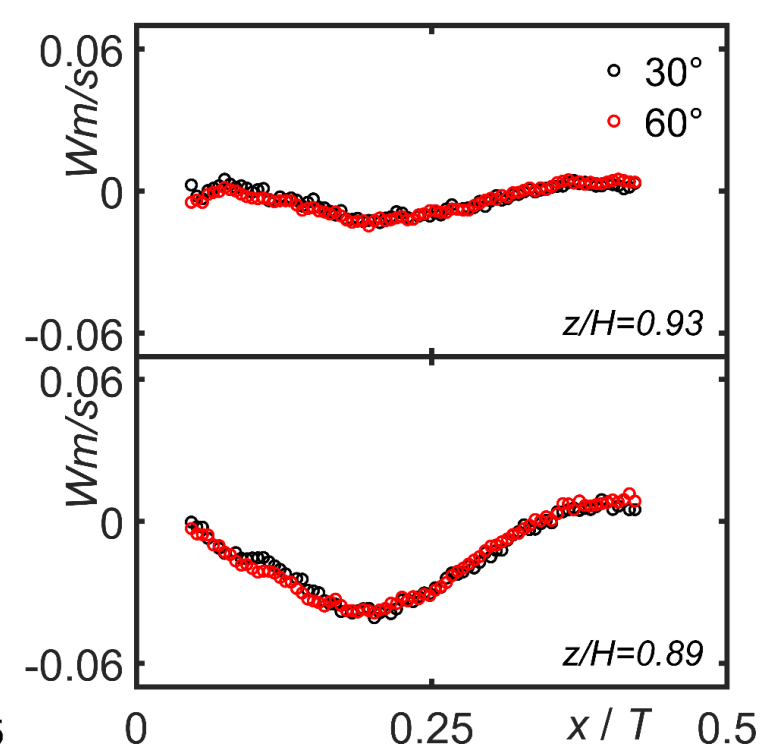

(b)

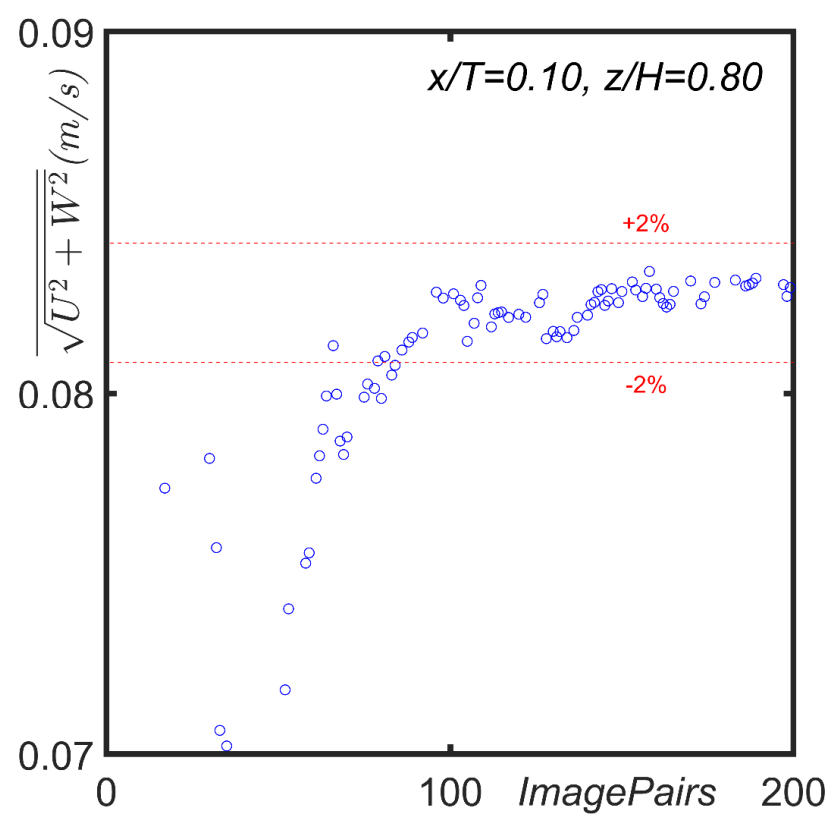

(d)

Fig. 4. (a): Mean velocity field of the continuous phase for two impeller angles $\theta=30^{\circ}$ and $\theta=60^{\circ}$. (b): axial velocity profiles for two impeller angles $\theta=30^{\circ}$ and $\theta=60^{\circ}$ at two horizontal lines $z / H=0.89$ and $z / H=0.93$. Average velocity in the measurement plane as a function of image pairs at point $x / T=0.30, z / H=0.55$ (panel (c)) and at point $x / T=0.10$, $z / H=0.80$ (panel (d)). PIV data for $C=T / 2$ and $\Phi=4 \%$. 


\section{Figure 5}

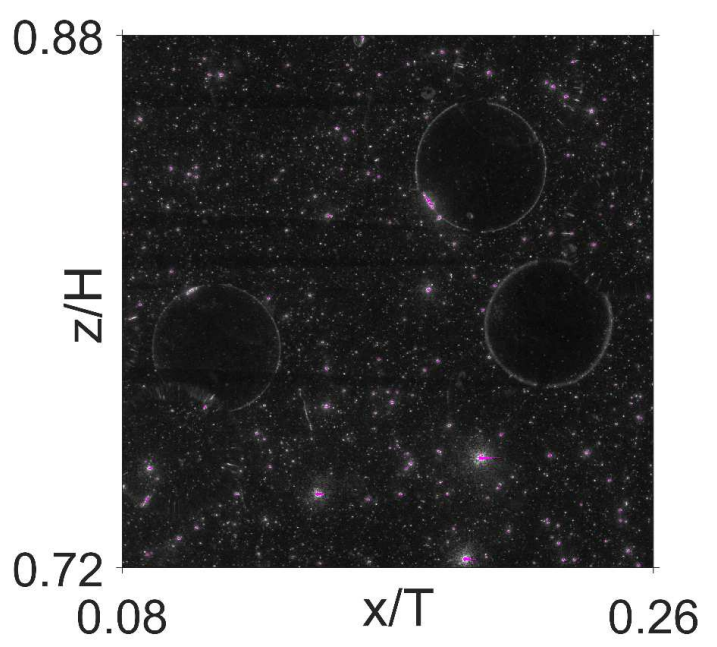

(a)

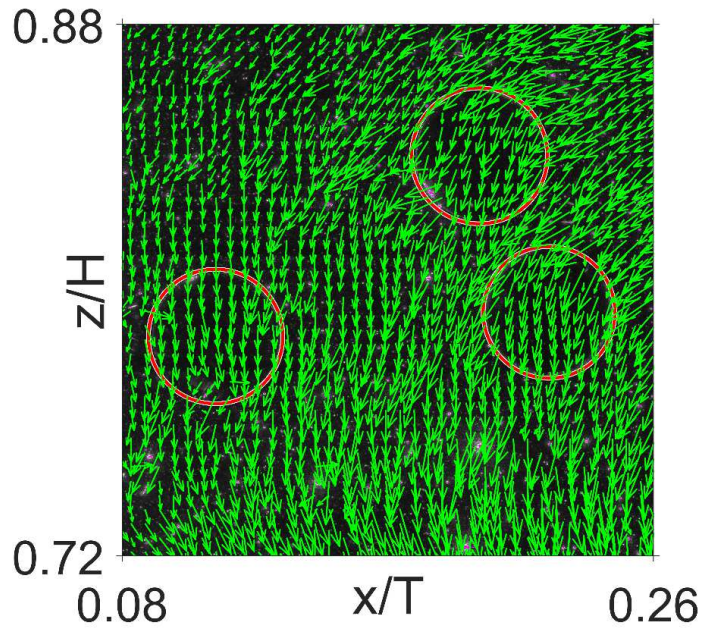

(c)

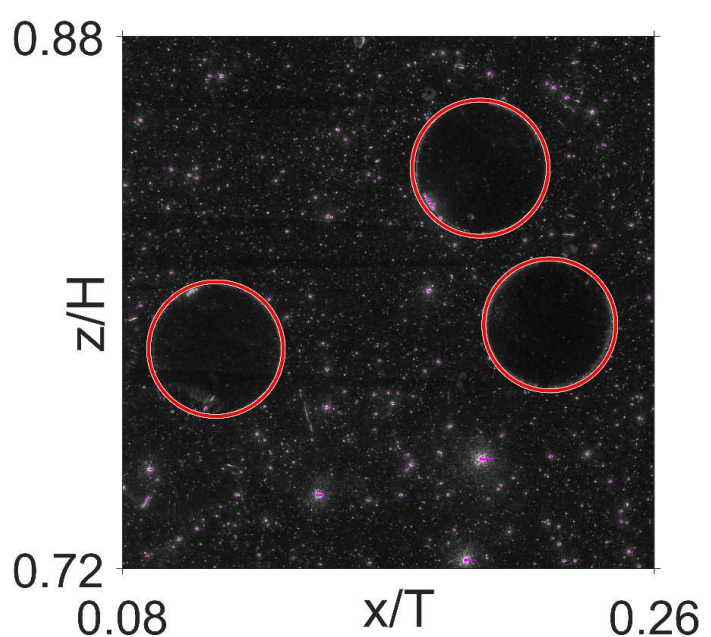

(b)

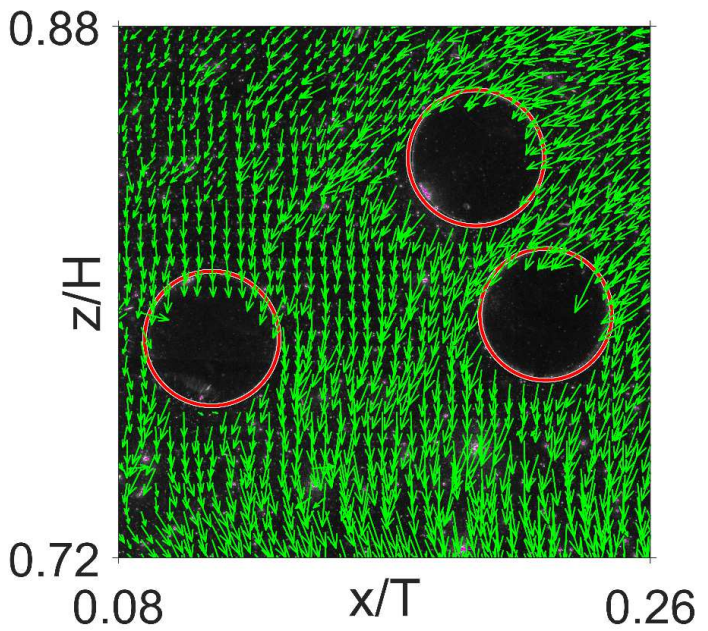

(d)

Fig. 5. Particle boundary detection and deletion of velocity vectors inside the boundaries. (a) PIV raw image; (b) PIV images with particle detection; (c) instantaneous local velocity vectors without post-processing; (d) instantaneous local velocity vectors after deleting the vectors inside the particles. 


\section{Figure 6}

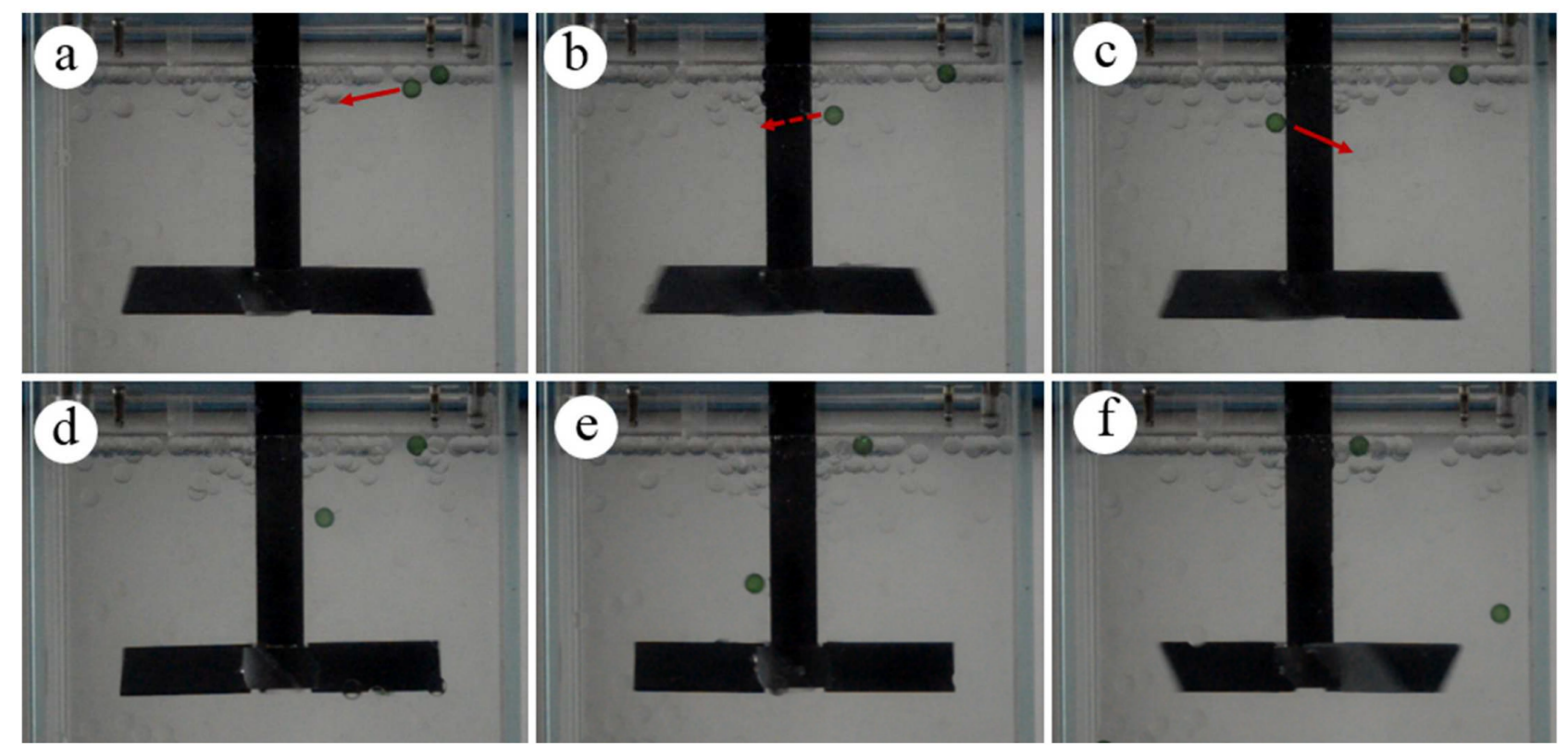

Fig. 6. The drawdown process of a green floating particle at an overall solids volume fraction $\Phi=1 \%$ (case 1 ). 


\section{Figure 7}

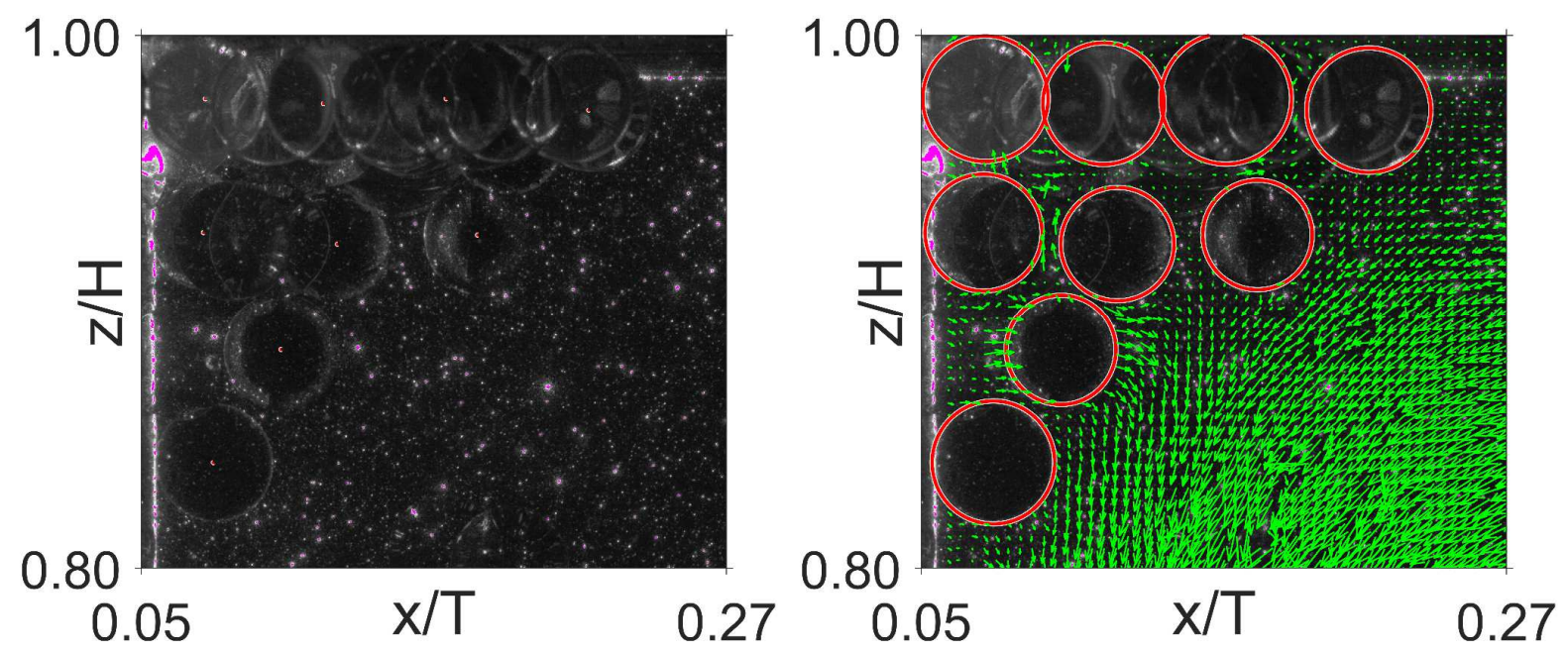

Fig. 7. Drawdown pattern of floating particles near the shaft (left) and the lid (top) and velocity vectors of the surrounding flow. 


\section{Figure 8}
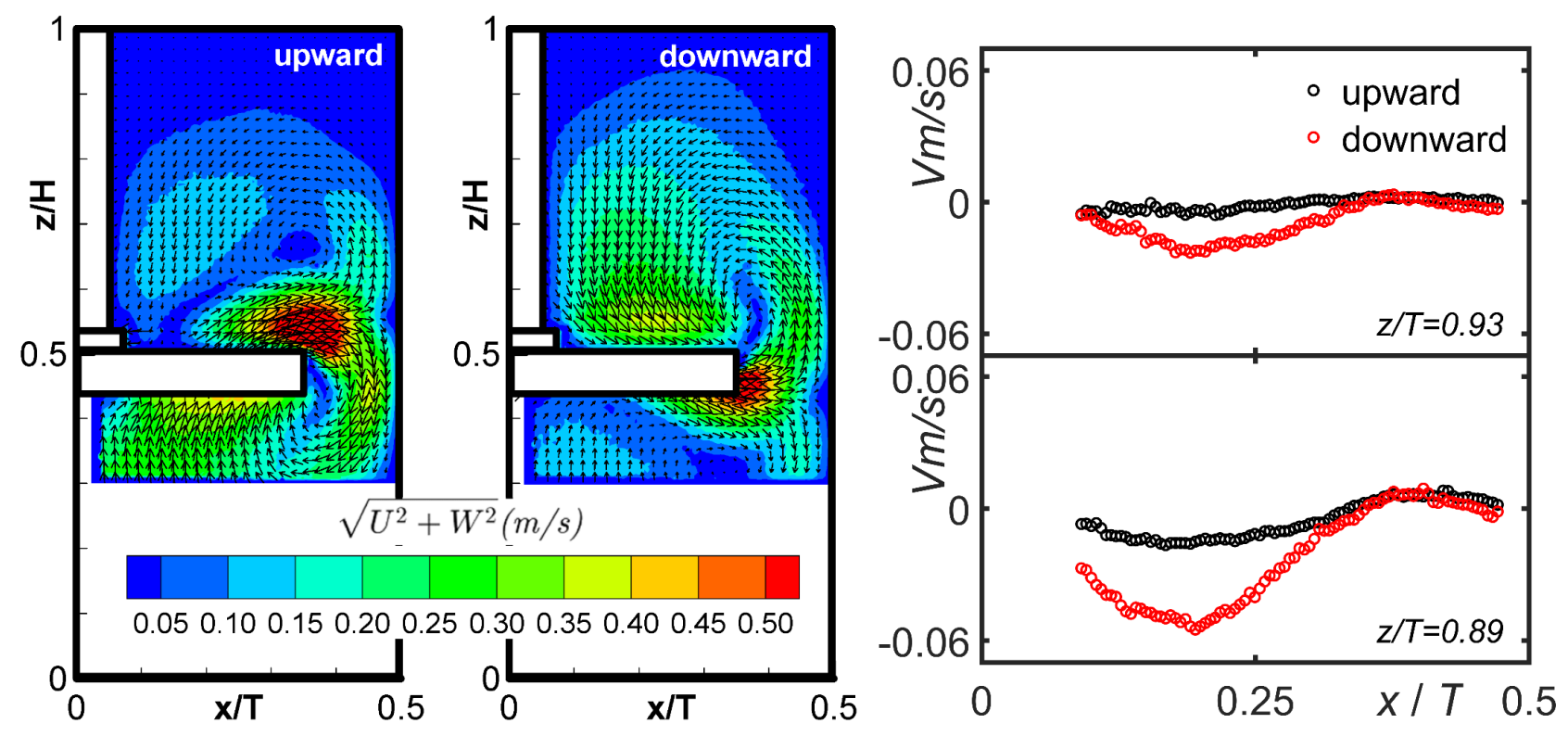

Fig. 8. Mean velocity field of the continuous phase for the upward (left panel, $N_{\mathrm{id}}=183.1 \mathrm{rpm}$ ) and downward (middle panel, $N_{\mathrm{jd}}=158.9 \mathrm{rpm}$ ) impeller pumping modes and axial velocity profiles for the two pumping modes at two horizontal lines $z / H=0.89$ and $z / H=0.93$ (right panel). $C=T / 2, \Phi=2 \%$, PIV data. 


\section{Figure 9}
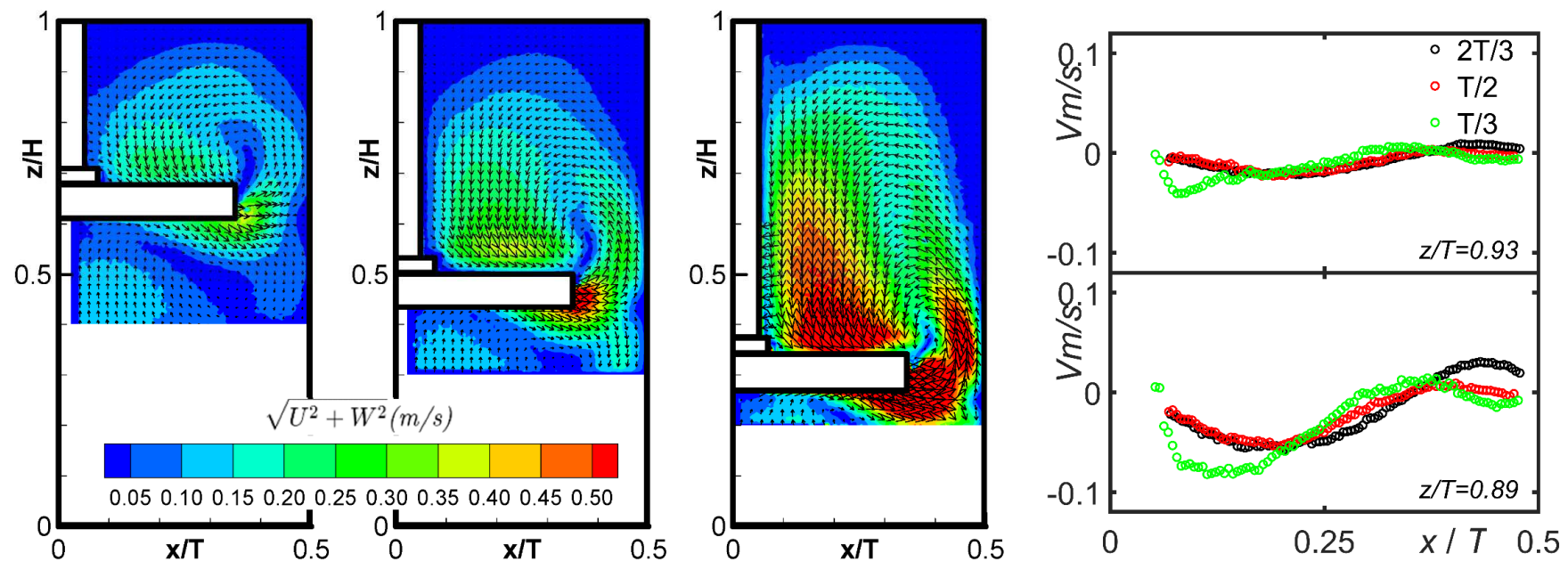

Fig. 9. Mean velocity field of the continuous phase for three impeller clearances (left three panels, the just drawdown speeds are $121.3 \mathrm{rpm}, 158.9 \mathrm{rpm}$, and $285.5 \mathrm{rpm}$ for $C=2 / 3 T, T / 2$, and $T / 3$, respectively) and axial velocity profiles for different impeller clearances at two horizontal lines $z / H=0.89$ and $z / H=0.93$ (very right panel). $\Phi=2 \%$, PIV data, downward pumping mode. 


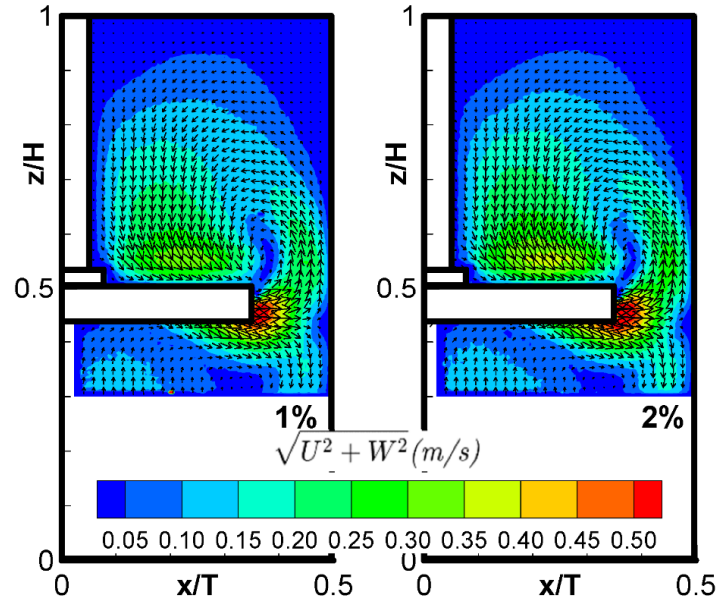

(a)

(b)

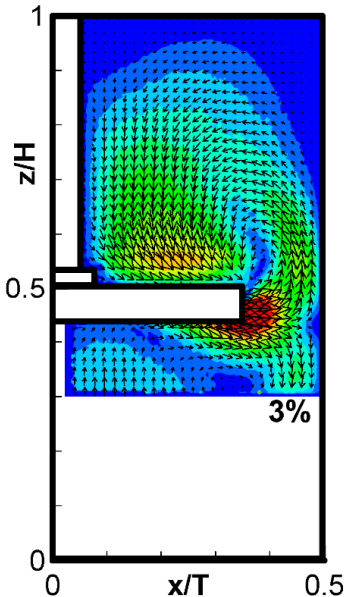

(c)

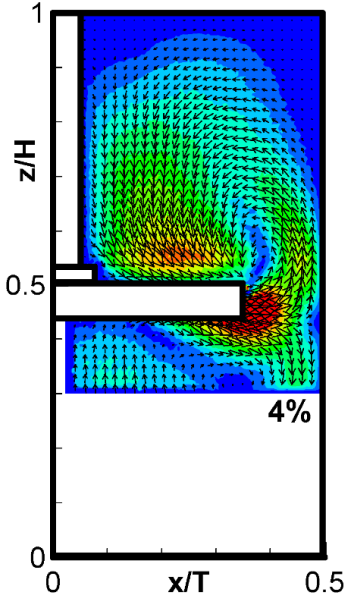

(d)

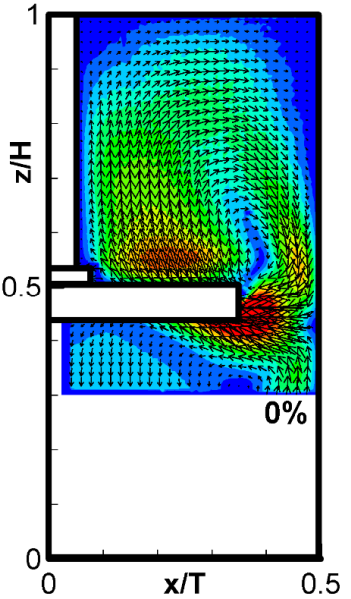

(e)

Fig. 10. Mean velocity field of the continuous phase for four solids concentrations measured by PIV with downward impeller pumping mode and $\mathrm{C}=\mathrm{T} / 2$. The just drawdown speeds are $143.8 \mathrm{rpm}, 158.9 \mathrm{rpm}, 182.7 \mathrm{rpm}$, and $197.4 \mathrm{rpm}$ for $\Phi=1 \%, 2 \%, 3 \%$, and $4 \%$, respectively. A single-phase flow field with $N=197.4 \mathrm{rpm}$ is presented for reference. 

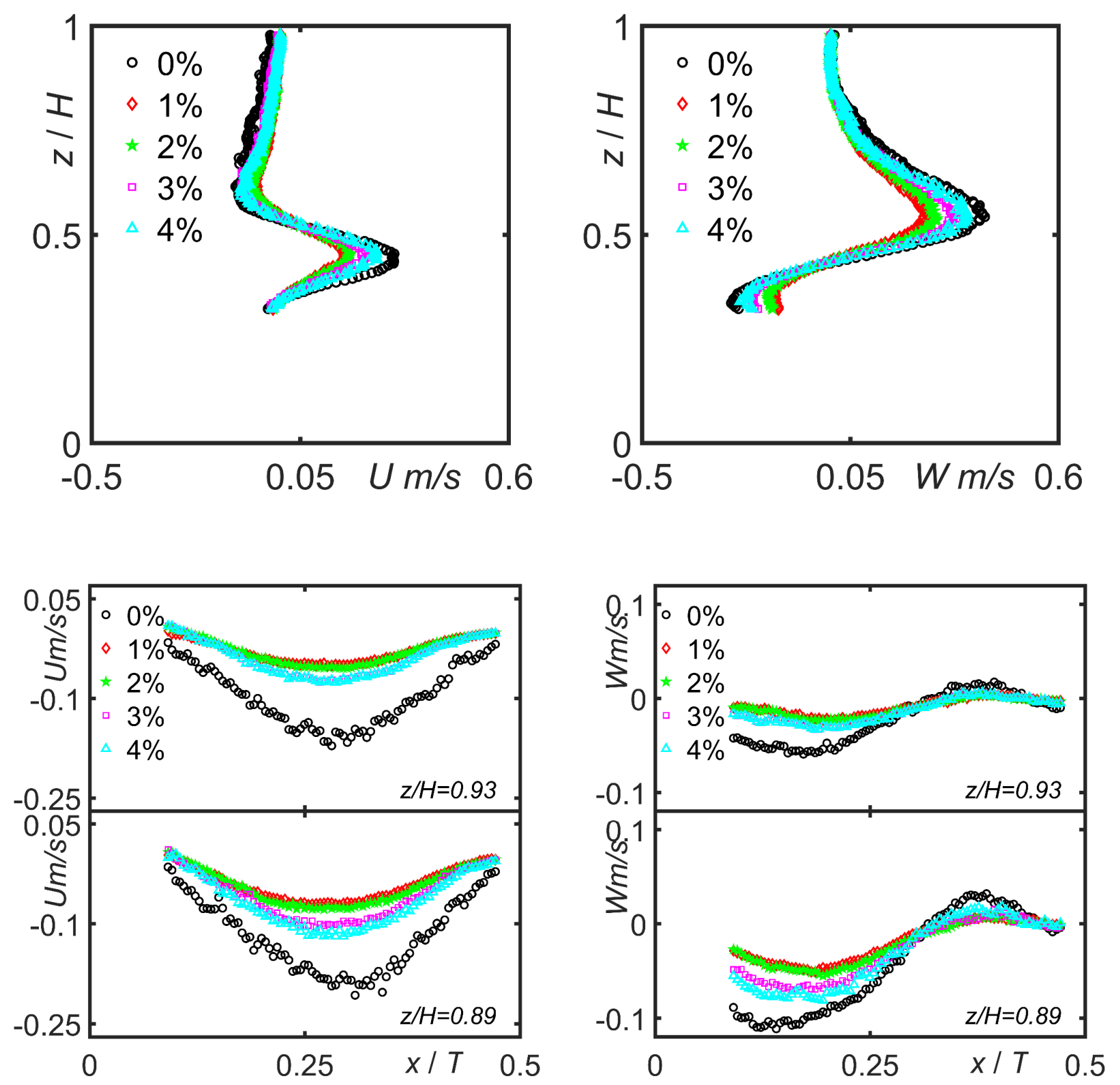

Fig. 11. Mean radial (left column) and axial (right column) velocity profiles for different solids concentrations at a vertical line $x / T=0.45$ (top row) and at two horizontal lines $z / H=0.89$ and $z / H=0.93$ (bottom row) measured by PIV with downward impeller pumping mode and $C=T / 2$. The just drawdown speeds are $143.8 \mathrm{rpm}, 158.9 \mathrm{rpm}, 182.7$ rpm, and $197.4 \mathrm{rpm}$ for $\Phi=1 \%, 2 \%, 3 \%$, and $4 \%$, respectively. The impeller speed for $\Phi=0 \%$ (single phase) is $N=197.4$ rpm. 
Figure 12

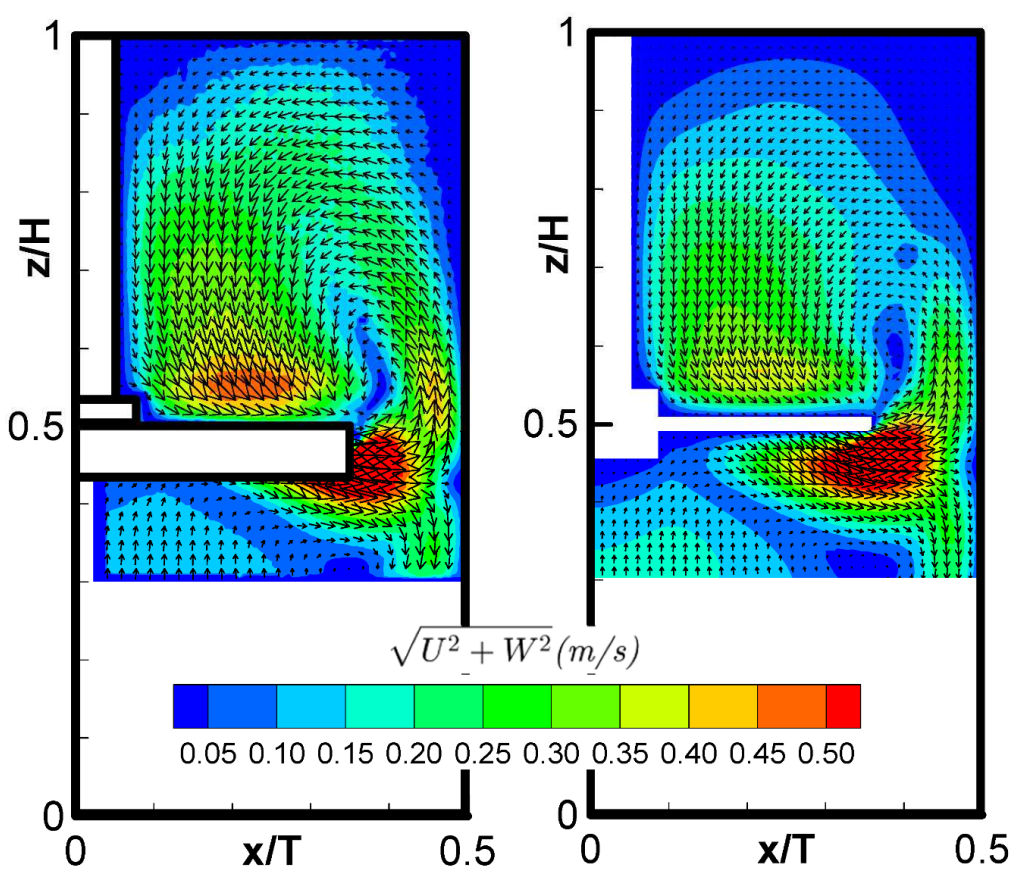

Fig. 12. Experimental (left) and simulated (right) mean velocity field of the single-phase flow with downward impeller pumping mode, $C=T / 2, \theta=0^{\circ}$, and $N=197.4 \mathrm{rpm}$. 
Figure 13
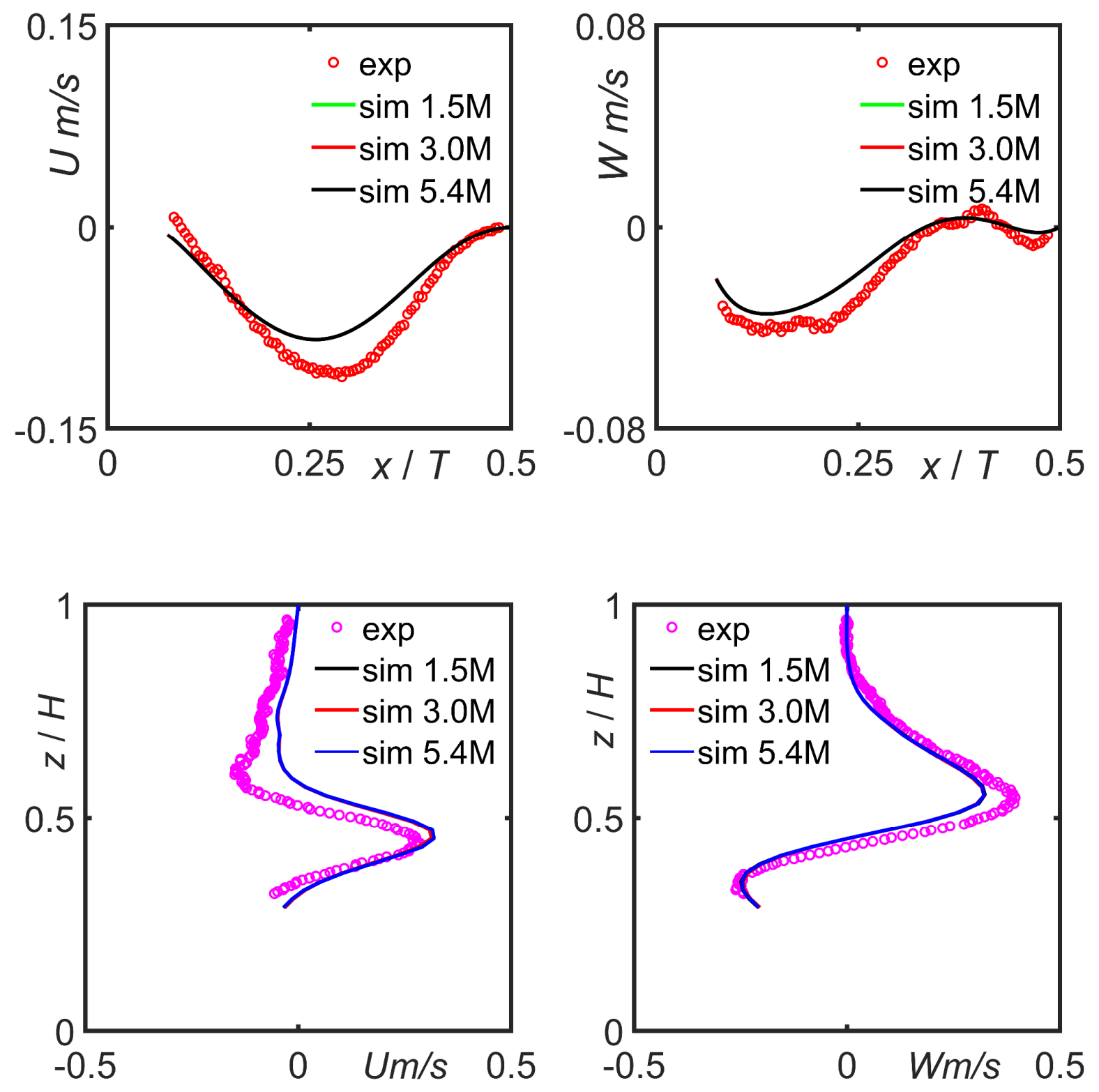

Fig. 13. Mean radial (left column) and axial (right column) velocity profiles at a horizontal line $z / H=0.93$ (top row) and a vertical line $x / T=0.45$ (bottom row). The simulated results with $1.5,3.0$, and 5.4 million grids are compared. The impeller is downward pumping with $C=T / 2$ and $N=197.4 \mathrm{rpm}$. Single-phase systems. 
Figure 14

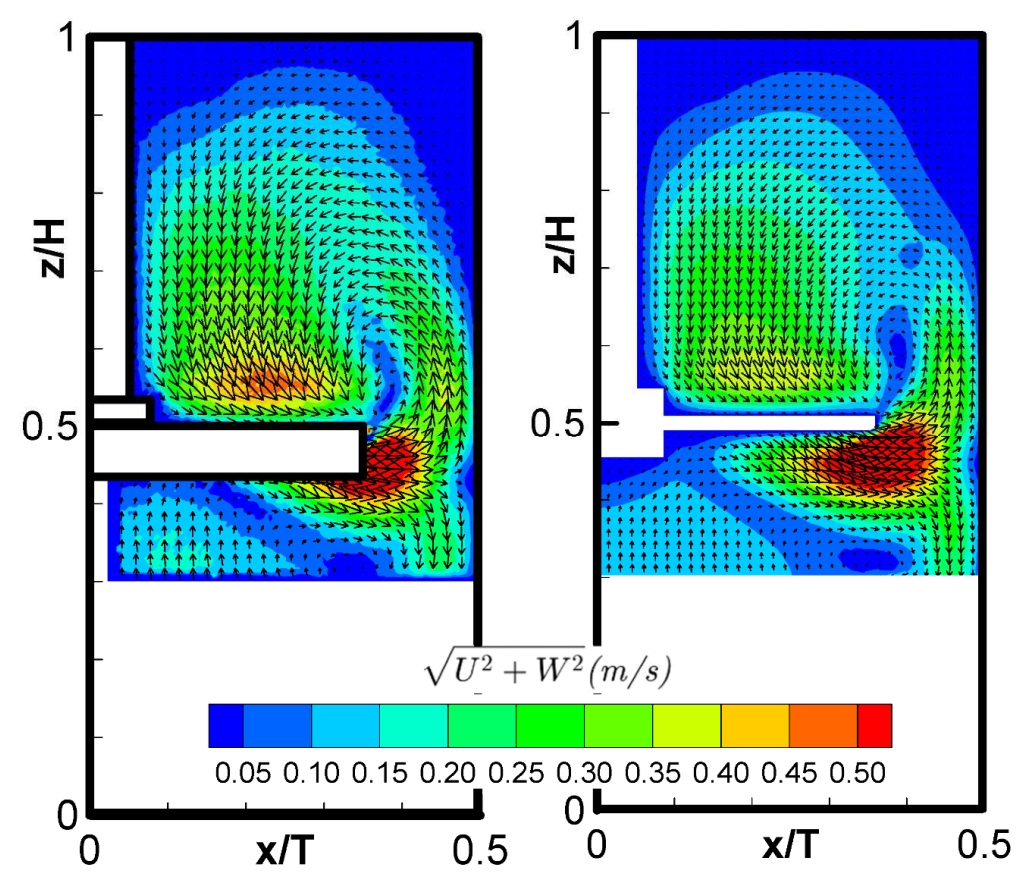

Fig. 14. Experimental (left) and simulated (right) mean velocity field of the continuous phase with downward impeller pumping mode, $C=T / 2, \Phi=4 \%, \theta=0^{\circ}$, and $N_{\mathrm{jd}}=197.4 \mathrm{rpm}$. 
Figure 15
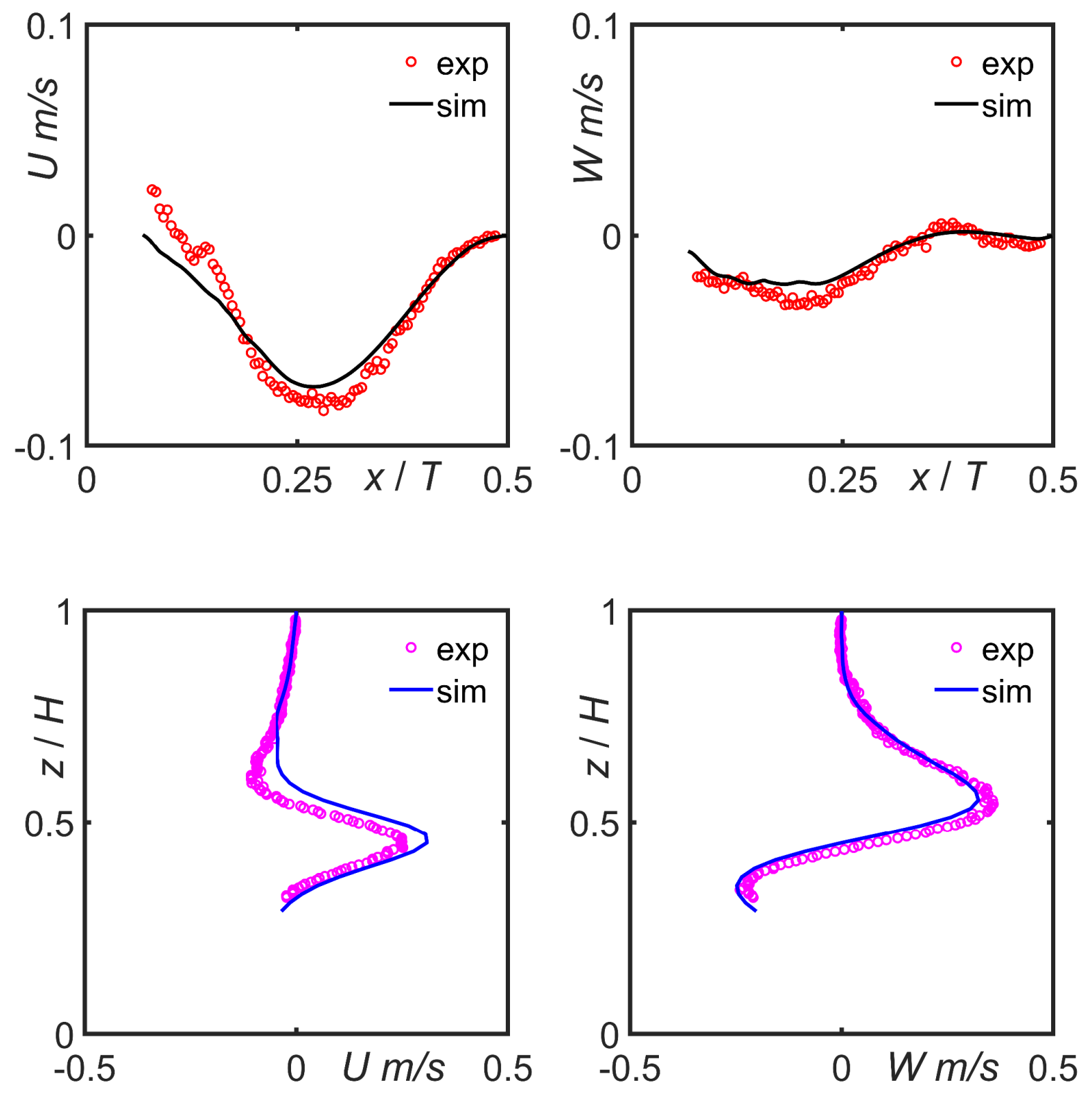

Fig. 15. Mean radial (left column) and axial (right column) velocity profiles at a horizontal line $z / H=0.93$ (top row) and a vertical line $x / T=0.45$ (bottom row). The impeller is downward pumping with $C=T / 2, N_{\mathrm{jd}}=197.4 \mathrm{rpm}$, and $\Phi=4 \%$. 


\section{Figure 16}
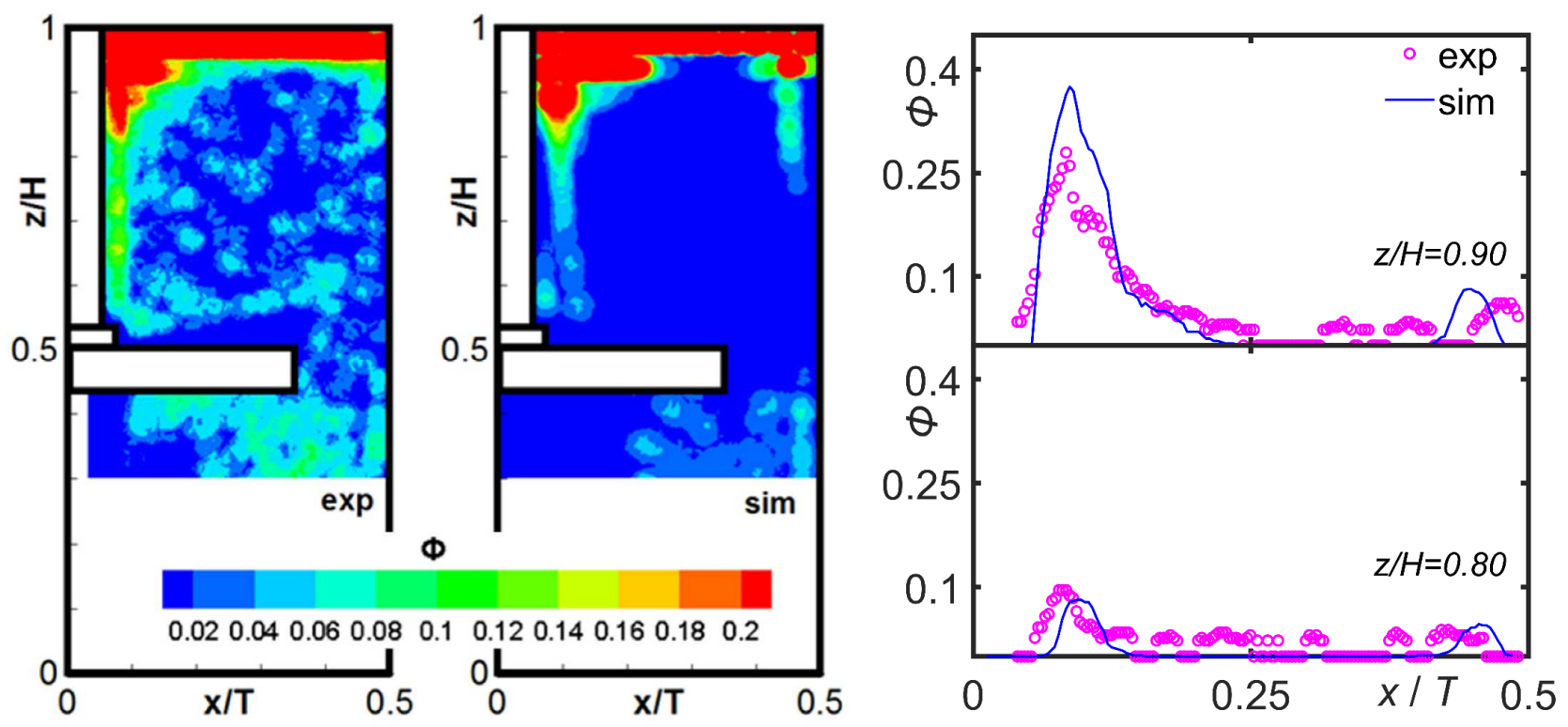

Fig. 16. Left and middle panels: experimental and simulated averaged solid volume fraction contours with downward impeller pumping mode, $C=T / 2, \Phi=4 \%, \theta=0^{\circ}$, and $N_{\mathrm{jd}}=197.4 \mathrm{rpm}$. Right panel: experimental and simulated averaged solid volume fraction profiles at two horizontal lines $z / H=0.9$ and $z / H=0.8$. 
Figure 17

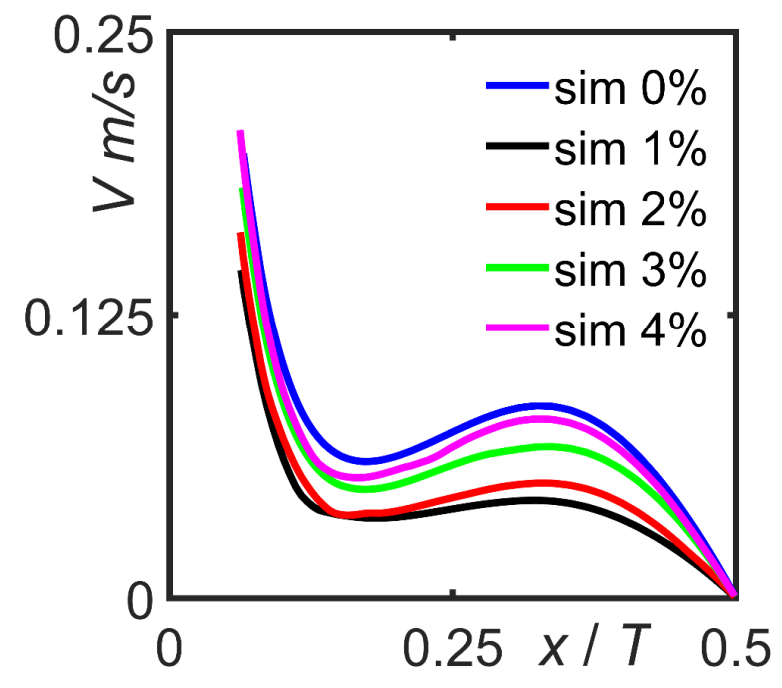

Fig. 17. The simulated tangential velocity profiles at a horizontal line $z / H=0.93$ for different solid concentrations. 


\section{Figure 18}
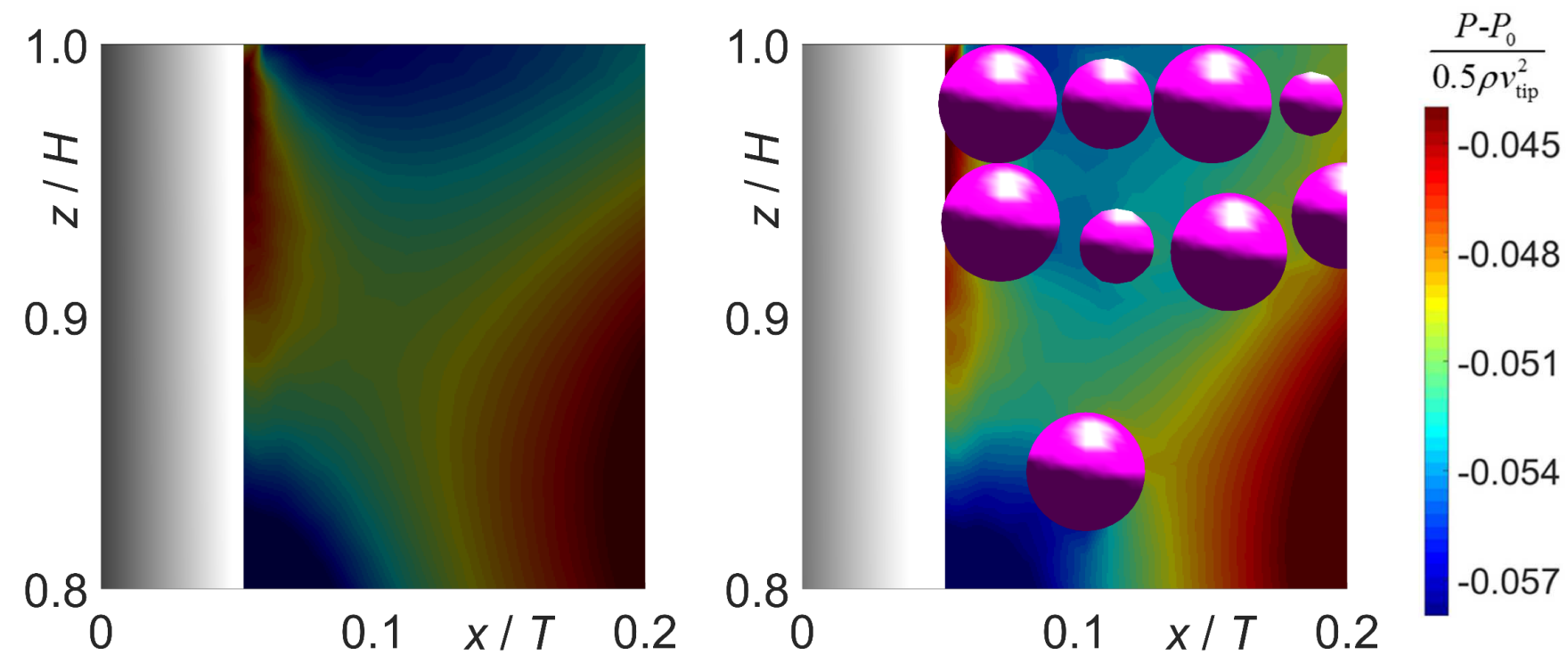

Fig. 18. Pressure field contours for $\Phi=0 \%$ (left) and $\Phi=4 \%$ (right) with downward impeller pumping mode, $C=T / 2$, $\theta=0^{\circ}$, and $N_{\mathrm{jd}}=197.4 \mathrm{rpm}$. 


\section{Figure 19}
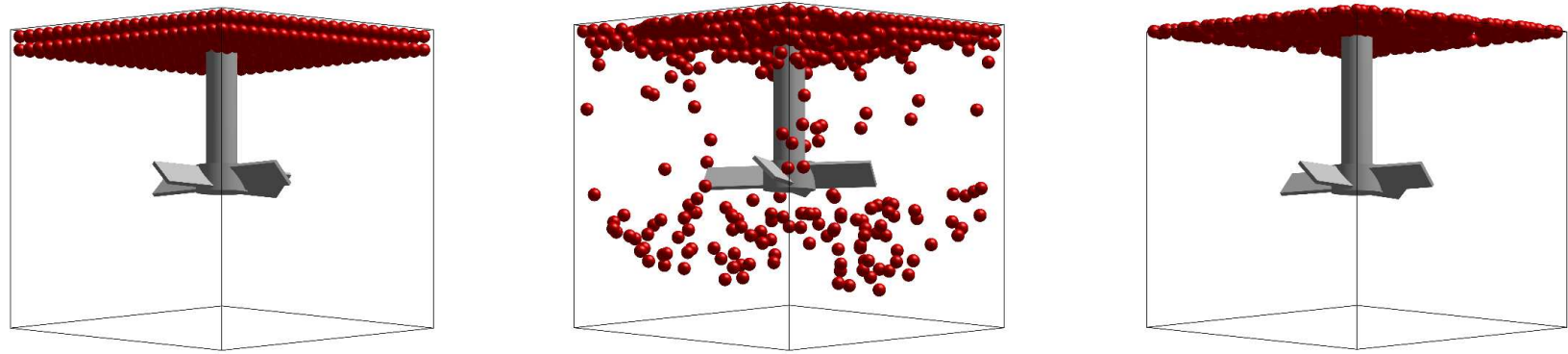

Fig. 19. The initial positions of particles (left) and the instantaneous particle positons simulated by the

Eulerian-Lagrangian approach with the DPM-DEM model (middle) and without the DEM model (right) at just drawdown condition with $\Phi=4 \%$. 